\title{
Speculative Linearizability
}

\author{
Rachid Guerraoui Viktor Kuncak Giuliano Losa \\ School of Computer and Communication Sciences \\ Swiss Federal Institute of Technology Lausanne (EPFL) \\ rachid.guerraoui@epfl.ch viktor.kuncak@epfl.ch giuliano.losa@epfl.ch
}

\begin{abstract}
Linearizability is a key design methodology for reasoning about implementations of concurrent abstract data types in both shared memory and message passing systems. It provides the illusion that operations execute sequentially and fault-free, despite the asynchrony and faults inherent to a concurrent system, especially a distributed one. A key property of linearizability is inter-object composability: a system composed of linearizable objects is itself linearizable. However, devising linearizable objects is very difficult, requiring complex algorithms to work correctly under general circumstances, and often resulting in bad average-case behavior. Concurrent algorithm designers therefore resort to speculation: optimizing algorithms to handle common scenarios more efficiently. The outcome are even more complex protocols, for which it is no longer tractable to prove their correctness.

To simplify the design of efficient yet robust linearizable protocols, we propose a new notion: speculative linearizability. This property is as general as linearizability, yet it allows intra-object composability: the correctness of independent protocol phases implies the correctness of their composition. In particular, it allows the designer to focus solely on the proof of an optimization and derive the correctness of the overall protocol from the correctness of the existing, non-optimized one.

Our notion of protocol phases allows processes to independently switch from one phase to another, without requiring them to reach agreement to determine the change of a phase. To illustrate the applicability of our methodology, we show how examples of speculative algorithms for shared memory and asynchronous message passing naturally fit into our framework.

We rigorously define speculative linearizability and prove our intra-object composition theorem in a trace-based as well as an automaton-based model. To obtain a further degree of confidence, we also formalize and mechanically check the theorem in the automaton-based model, using the I/O automata framework within the Isabelle interactive proof assistant. We expect our framework to enable, for the first time, scalable specifications and mechanical proofs of speculative implementations of linearizable objects.
\end{abstract}

\section{Introduction}

The correctness of a system of processes communicating through linearizable objects $[12,17,18]$ reduces to the correctness of the

Permission to make digital or hard copies of all or part of this work for personal or classroom use is granted without fee provided that copies are not made or distributed for profit or commercial advantage and that copies bear this notice and the full citation on the first page. To copy otherwise, to republish, to post on servers or to redistribute to lists, requires prior specific permission and/or a fee.

PLDI'12, June 11-16, 2012, Beijing, China.

Copyright (C) 2012 ACM 978-1-4503-1205-9/12/06. . \$10.00 sequential executions of that system. In other words, linearizability reduces the difficult problem of reasoning about concurrent data types to that of reasoning about sequential ones. In this sense, the use of linearizable objects greatly simplifies the construction of concurrent systems. At first glance, the design and implementation of linearizable objects themselves looks also simple. One can focus on each object independently, design the underlying linearizable algorithm, implement and test it, and then compose it with algorithms ensuring the linearizability of the other objects of the system. In short, linearizability is preserved by inter-object composition: a set of objects is linearizable if and only if each object is linearizable when considered independently of the others.

However, it is extremely difficult to devise efficient and robust implementations of a linearizable object, even when considered independently from the others. The difficulty stems from the following dilemma. On the one hand, achieving robustness in a concurrent system requires assuming that the scheduling of processes, communication, and faults is completely nondeterministic. The object does not know which execution is going to unfold but needs to deliver a response to each of its method invocations no matter the execution. The object has to prepare for all scenarios, using up its resources for that task. This conservative approach typically yields wait-free [11] but inefficient strategies. On the other hand, achieving efficiency requires speculating that only a small subset of executions is likely to occur [23]. This is practically appealing because a real system typically spends most of its time in a common case and seldom experiences periods of alternative executions. Should the object focus on a very small subset of executions, it would save resources by preparing only for that restricted subset. Typically, the common cases that are usually considered in practice are those where the system is synchronous, there is no failure, contention is not very high, a specific conditional branch is to be considered, etc. At a high level, a speculative system repeats the following steps:

1. Speculate about the likelihood of certain executions and choose a corresponding algorithm.

2. Initialize the chosen algorithm.

3. Monitor the algorithm to estimate if the speculation was accurate. If not, abort the algorithm and recover a consistent state.

Examples of speculation include the Ethernet protocol, where processes speculatively occupy a single-user communication medium before backing off if collision is detected, or branch prediction in microprocessors, where the processor speculates that a particular branch in the code will be taken before discarding its computation if this is not the case. More recent instances of speculation include optimistic replication [15] or adaptive mutual exclusion [14]. In fact, most practical concurrent systems are speculative. In general, speculative systems may choose between many different options, or speculation phases, in order to closely match a changing common case. 
To illustrate the challenges addressed in this work, consider a speculative algorithm that may switch between any two of $n$ speculation phases in a safe manner. That is, recovering a consistent state of the first phase and then initializing the second. Doing so in an ad-hoc manner poses two major scalability problems to the design process. First, there are $O\left(n^{2}\right)$ different switching cases that need to be carefully handled in order to preserve linearizability. Second, adding a new phase to an object composed of $n$ phases built ad-hoc may require deep changes to all of the $n$ existing phases.

The case of State Machine Replication [16] (abbreviated SMR), used to build robust linearizable object implementations, illustrates those problems. Non-speculative SMR algorithms like Paxos [7] or PBFT [6] are notoriously hard to understand. The formal correctness proof of Disk Paxos [13] took about 7000 lines. Only an informal proof, 35 pages long, of a simplified version of PBFT is known to the authors [5]. Speculative SMR protocols are even harder. For instance, the Zyzzyva [15] protocol combines PBFT with a fast path implemented by a simple agreement protocol. The fast path is more efficient than PBFT when there are no failures. In the advent of failures, the fast path cannot make progress and Zyzzyva falls back to executing PBFT. The ad-hoc composition of the fast path with PBFT required deep changes to both algorithms and resulted in an entanglement that is hardly understandable. Although PBFT had previously been widely tested [5], Zyzzyva suffered from fragile implementations and no correctness proof has ever been proposed. In fact, Zyzzyva, being restricted to two phases, is very fragile [24]. If the common case is not what is expected by the fast path one falls-back to PBFT, making the optimization useless. An adversary can easily weaken the system by always making it abort the fast path and go through the slow one. Introducing a new dimension of speculation might make the protocol more robust but would require a new ad-hoc composition, including an alternative fast-path, at a cost comparable to the effort needed to build Zyzzyva from scratch, namely a Dantean effort. Given the diversity of situations encountered in practice, we are convinced that this ad-hoc approach is simply intractable.

Contributions. We propose in this paper a framework for devising, reasoning about, and mechanically verifying effective and robust linearizable implementations of concurrent data types in a scalable way. In short, we show how speculative implementations of linearizable objects can be devised and analyzed in an incremental manner. One can focus on each dimension of speculation independently of the others. For example, a programmer can first devise an algorithm with no speculation, implement, test, and prove it correct, then augment it later with an optimization that speculates that a certain subset of executions is more likely to occur (say there is no failure). This optimization sub-algorithm is composed with the original one as is. Later, another programmer can consider other dimensions of speculations (say there is no contention or asynchrony) and add new phases, still without modifying the original implementation and proofs.

At the heart of our framework lies our new notion, speculative linearizability. Speculative linearizability encompasses the notion of switch actions, which makes it significantly richer than linearizability, yet it reduces to linearizability if these actions are ignored. Speculative linearizability augments classical linearizability with a new aspect of composition. Not only a system of concurrent objects can be considered correct if each of them is correct (interobject composition), but a set of algorithms implementing different speculation phases of the same object is correct if each of them is correct (intra-object composition). We express this new aspect through a new composition theorem which we state and prove. Intuitively, speculative linearizability captures the idea of safe and live abortability. An implementation can abort if the assumptions

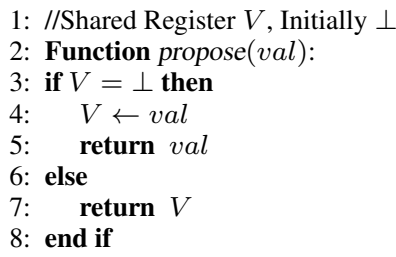

Figure 1. Consensus Specification

behind speculation reveals wrong. When it does abort, it does so in a safe manner, by preserving the consistency (linearizability) of the object state. Moreover, the abort is also performed in a live manner, because a new protocol phase can resume and make progress. Processes can switch independently from one phase to another, without the need to reach agreement. Our notion of speculative linearizability is itself based on a new definition of linearizability. This definition is interesting in its own right because it enables a more local form of reasoning than the original definition and it allows for repeated events, which are the norm in practice. We have proved that our new definition of linearizability is equivalent to the classical definition.

The generality of our framework and its Isabelle/HOL formalization [10] sets our work apart from state of the art in the area $[1,2,8]$ : we enable for the first time scalable mechanically-checked development of linearizable objects of arbitrary abstract data types.

Additional proofs and formalizations are available in $[9,10]$, as well as at http://lara.epfl.ch/w/slin.

\section{Putting Speculative Linearizability to Work}

In this section we provide intuition for speculative linearizability and its key property: intra-object composability. Our goal is to motivate correct-by-construction design of objects based on speculation phases. As an illustration, we present two speculative implementations of a consensus object. Each is composed of two speculation phases. The first applies to the message-passing computation model and the other to the shared-memory model. We analyze each phase of those speculative implementations in isolation and conclude, using the intra-object composition theorem, that their compositions are correct implementations of consensus.

\subsection{Message-Passing Consensus}

Our first example is a consensus implementation in a system composed of client and server processes which communicate by asynchronous message passing and which may crash at any point. Our goal is to implement consensus among the clients. Notable use cases of consensus in message-passing systems include Google's Chubby distributed lock service [4] and the Gaios reliable data store [3].

Consensus allows a set of clients to agree on a common value. Clients each propose a value and should receive a common decision value taken among their proposals. We consider implementations that offer an invocation function propose (value) to each of its clients. The invocation propose $(\mathrm{val})$ returns another value indicating the common decision. When a process returns from propose(value) with a value $d$, we say the it decides value $d$. An algorithm is linearizable with respect to the consensus data type if all calls to propose $(\mathrm{val})$ appear as if they executed atomically the algorithm in Figure 1 at some point after their invocation (we assume that proposal are different from $\perp$ ).

In an asynchronous message-passing system where processes may crash, there is no component which can reliably hold state. Hence, some crucial data may become unavailable due to the failure of a process. For example, a process may decide some value and 
then crash before informing any other process of its decision. In this case other processes have no way to know which value the crashed process decided and they cannot decide without risking to violate consensus.

Paxos [7] is an algorithm that implements consensus if less than half of the servers may fail. Paxos has a minimum latency of 3 message delays. However, suppose that executions are fault free and contention free (i.e. the time intervals delimited by corresponding invocations and responses do not overlap). Under this assumption, very simple algorithms can solve consensus much faster than Paxos, which still has a minimum latency of 3 message delays.

We would like to optimize Paxos for the fault-free and contention-free case. This could be done ad-hoc by adding a fast path inside Paxos. However, Paxos is a very intricate algorithm: the proof of Disk Paxos [7], a variant of Paxos, is 7000 lines long [13]. Adding a fast path to Paxos would require re-examining the correctness of the entire algorithm, because the fast path is interleaved with executions of the others parts. The entire proof would, in principle, need to be rewritten.

Our framework allows us to optimize Paxos without directly modifying the original algorithm, and enables reasoning about the correctness of the optimization independently of the basic Paxos itself. To use our framework, we wrap Paxos in a simple interface that allows composition with other speculation phases. This requires adding a trivial level of indirection and makes Paxos a speculation phase in its own right. Speculative linearizability ensures that any speculatively linearizable speculation phase can be composed with the Paxos speculation phase to form a correct implementation of consensus. Moreover, speculative linearizability, instantiated for consensus, is a property independent of Paxos. Therefore, from the point of view of algorithm designers, speculative linearizability hides the internal complexity of Paxos.

In this example we compose Paxos with the Quorum speculation phase. Quorum manages to decide on the value in only 2 message delays, whenever there is neither contention nor faults. If faults or contention happen, Quorum cannot decide and instead passes control to Paxos. In this case, we say that Quorum switches to Paxos. From then on, Paxos takes over the execution and can decide as long as less than half of the processes are faulty. To highlight that Paxos is used as a backup when optimization by Quorum is not possible, we call the Paxos speculation phase Backup. Using speculative linearizability, we will prove that the composition of Quorum and Backup is linearizable by reasoning about Quorum in isolation and by reusing an existing proof of Paxos.

By combining Quorum and Backup we obtain a system that is optimized for contention-free and fault-free loads while still remaining correct in all other conditions under which the Backup is correct. Such an approach has proved empirically successful in recent work [8]; the present paper provides the underlying theoretical foundations for such approaches.

We now describe more precisely the Quorum and Backup speculation phases. Their interface is that of a consensus object, as defined above, augmented with a switch-to-backup $(s v)$ call. The switch-to-backup $(s v)$ call is used by a client to switch from the Quorum phase to Backup, i.e. switch-to-backup $(s v)$ is a procedure of Backup which may be called by a client executing Quorum. The $s v$ parameter is called a switch value. A client calling switch-to-backup $(s v)$ transfer its pending invocation to Backup and provides $s v$ as an indication to Backup on how to take over Quorum's execution.

Informally, the Quorum algorithm works as follows:

- Upon $\operatorname{propose}(v)$, a client $c$ broadcasts its proposal to all server processes and waits for accept messages. It also stores $v$ in local variable proposal $l_{c}$ of its memory and starts a local timer $t_{c}$.
- When a server process receives a proposal $v$ from a client $c$ :

- If it has not sent any accept message, it sends an accept message $\operatorname{accept}(v)$ to $c$.

- If it has already sent an accept message $\operatorname{accept}\left(v^{\prime}\right)$, it sends $\operatorname{accept}\left(v^{\prime}\right)$ to $c$.

- If a client receives two different accept messages, it switches to Backup by calling and returning switch-to-backup $\left(\right.$ proposal $\left._{c}\right)$.

- If a client receives the same accept messages accept $(v)$ from all the servers, then it decides $v$.

- If timer $t_{c}$ expires, then $c$ waits for at least one message accept $\left(v^{\prime}\right)$ from a server, or selects one $v^{\prime}$ if it has some messages of the form accept $\left(v^{\prime}\right)$ already. It then switches to Backup by calling switch-to-backup $\left(v^{\prime}\right)$.

The Backup phase is Lamport's Paxos algorithm where clients have the role of proposers and learners, while servers have the role of acceptors. Backup treats the switch calls from Quorum as regular proposals. In other words, upon a call switch-to-backup $(v)$, a client process proposes the value $v$ to the Paxos algorithm.

By composing Quorum and Backup we obtain a linearizable implementation of consensus that is optimized for crash-free and contention-free workloads. This new optimized implementation of consensus was obtained by composing a black-box implementation of Paxos with a much simpler protocol, Quorum, which makes progress only under particular conditions.

We next uncover the principles behind this approach and we will show the correctness of our optimized protocol by reasoning about each speculation phase in isolation, independently of each other. Thanks to our modular approach, we will be able to rely the existing proof of Paxos [13] to show the correctness of Backup with minor effort.

\subsection{Linearizability}

Before explaining speculative linearizability, we briefly review linearizability itself: we provide both its classical definition and our new formalization.

We consider a set of concurrent clients accessing an object through invocation procedures whose execution ends by returning a response, as in the consensus examples above. We assume that each client is sequential. In other words, a client does not invoke the object before having returned from its preceding invocation.

An object is accessed sequentially if every response to an invocation immediately follows the invocation. There must be no other response or invocation in between them. For example, the specification of a consensus object states that all processes return the same decision value and that this value must have been proposed by some process before it can be returned. Hence, in a sequential execution, the first proposed value must be returned to every process. Note that this corresponds to executing sequentially the algorithm in Figure 1: The first process executing will impose its value to all others.

We consider only deterministic objects. Therefore, in the sequential case, the response to a given invocation is determined by the sequence of past invocations that the object received. Therefore, we represent a sequential execution by its subsequence containing only and all invocations in the execution. We call such sequences of invocations histories.

When accessed concurrently, invocations and responses of different processes may be arbitrarily interleaved. The sequences of invocations and responses that may be observed at the interface of an object that is accessed concurrently are called traces. The occurrence of an invocation or a response in a trace is called an event, or action.

Linearizability is defined with respect to a sequential specification, which is a description of the allowed behaviors of the object 
when it is accessed sequentially. Intuitively, a trace is linearizable if each invocation appears to take effect at a single point in time after the invocation call and before the corresponding response. This intuitive definition can be restated as follows: A trace $t$ is linearizable if we can associate, to each response $r$ returned by a client $c$, a history $h$ (called commit history) such that:

1 . The response $r$ is the response obtained in the sequential execution represented by history $h$.

2. All invocations in the history $h$ are invoked in the trace $t$ before the response $r$ is returned.

3. The history $h$ ends with the last invocation of client $c$.

4. For all commit histories $h_{1}$ and $h_{2}$, either $h_{1}$ is a prefix of $h_{2}$ or $h_{2}$ is a prefix of $h_{1}$.

This definition of linearizability is stated precisely in Section 4. In the terminology of Section 4, condition 1 above says that history $h$ explains response $r$. Condition 1 is specific to a particular abstract data type. Conditions 2 and 3 correspond to Validity, and 4 to Commit Order. They do not depend on the particular ADT considered. We have proved [9] that this definition is equivalent to the original definition of linearizability [12].

As an example, consider a trace of a consensus object such that client $c_{1}$ proposes value $v_{1}$, then client $c_{2}$ proposes value $v_{2}$, then client $c_{2}$ returns $v_{2}$, and finally client $c_{1}$ returns $v_{2}$. This execution is linearizable because the returned values are as if the invocation of client $c_{2}$ was executed atomically before the invocation of client $c_{1}$. To show this, associate the history $\left[\operatorname{propose}\left(v_{2}\right)\right]$ to the response of $c_{2}$ and the history $\left[\operatorname{propose}\left(v_{2}\right), \operatorname{propose}\left(v_{1}\right)\right]$ to the response of $c_{1}$. It is easy to check that all four conditions above are satisfied. Notably, when applied sequentially to a consensus object, both histories lead to response $v_{2}$. By the definition above, the trace is linearizable. Examples of traces that are not linearizable include:

[ $c_{1}$ proposes $v_{1}, c_{2}$ proposes $v_{2}, c_{1}$ decides $v_{1}, c_{2}$ decides $\left.v_{2}\right]$, as well as:

$\left[c_{1}\right.$ proposes $v_{1}, c_{1}$ decides $v_{2}, c_{2}$ proposes $v_{2}, c_{2}$ decides $\left.v_{2}\right]$.

\subsection{Speculative Linearizability}

We now consider modular implementations composed of two speculation phases (the definition generalizes to any number of phases). As in the composition of the Quorum and Backup algorithms, each client process starts by executing the first phase and may switch to the second phase using a procedure call, providing an argument that we call a switch value along with its pending invocation. In the examples of this section we omit the pending invocation from switch calls for the sake of conciseness.

We would like to reason about each speculation phase in isolation. For this purpose, we require that the switch values provided by the clients when changing phase be the only information passed from one speculation phase to the other. No side effects are permitted across speculation phases. Speculation phases are thus separated by a clear interface: a call to the phase's switch procedure with a switch value and a pending invocation as arguments.

Speculative linearizability is a property of speculation phases that relates the switch events and the invocations received by the speculation phase to the responses and switch events produced by the same phase. Moreover, no assumption other that trivial wellformedness conditions is made on the received switch events and invocations. This allows us to check that a phase is speculatively linearizable by reasoning about the phase independently of other phases it might be composed with.

Speculative linearizability is an extension of linearizability to traces that contain switch calls. In the definition of linearizability stated above, we associated to each response returned by a client a history of inputs with certain properties. In the same spirit, in addition to associating histories to responses returned, we now associate to each switch call in the trace a history of inputs. With respect to the first phase, we call these histories abort histories. With respect to the second phase, we call them init histories.

Such histories associated to switch calls represent possible linearizations of the execution of the first speculation phase. The idea is that, with the knowledge of how the execution of the first phase was linearized, the second phase can continue execution without violating linearizability. In order for a speculation phase to deduce a possible current linearization from a switch value, we suppose that all speculation phases agree on a common mapping from switch values to histories. We denote this mapping by $r_{\text {init }}$.

Depending on the particular data type being implemented, there are some sets of histories whose members all bring the system in the same logical state. In other words, the response to a new invocation is independent of which history of such a set was executed before. We call histories in such sets equivalent with respect to the data type. Consider our consensus example. Any set of histories whose members start with the same proposal is a set of equivalent histories: because the first proposal is the decision value, any new invocation will return the first proposed value. Processes invoking the object at a point after such a history has been executed cannot tell which history in the set actually happened. Because of this observation, we require that speculation phases agree on a mapping from switch values to sets of equivalent histories, as opposed to a single history. This allows us to use a compact representation of histories while ensuring that enough information is available for the second phase to take over the execution.

Two other principles, fault tolerance and avoidance of unnecessary synchronization, influenced our definition. For fault tolerance, we do not rely on just one client passing a single switch value. For efficiency reasons, we avoid the requirement that all clients switch with the same value because it would imply solving a potentially expensive agreement problem. Hence, the switch values of different clients may be different and no single switch call determines the execution of the second phase.

Applied to the first phase, speculative linearizability requires that the first phase be linearizable. Moreover, for all traces of the first phase, it must be possible to associate histories to the switch events so that each such history is a possible linearization of the trace up to the considered event and each association from switch event to history respects the common mapping that phases agree on. Finally, the longest common prefix of all abort histories must be a full linearization of the trace of the first phase. The last two requirements correspond to the Abort Order property of Section 5.

Applied to the second phase, speculative linearizability says that for all traces $t$ of the second phase, for all possible associations of histories to switch values that respect the common mapping agreed on, by concatenating $t$ to the trace represented by the longest common prefix of all init histories and by replacing switch calls with the pending invocation they contain, one should obtain a linearizable trace. This corresponds to the Init Order property of Section 5.

Having defined the new concept of speculative linearizability, we will show that the composition of any number of speculatively linearizable phases is a linearizable algorithm. This result is a corollary of our intra-object composition theorem, which states that the composition of two speculation phases is itself a speculation phase.

\subsection{Applying Speculative Linearizability to Quorum+Backup}

We next show that our message-passing speculation phases, Quorum and Backup, satisfy speculative linearizability. As explained above, we view the values passed when switching from Quorum to Backup as representing sets of histories equivalent to some lin- 
earization of Quorum's execution. To this end we assumed that speculation phases agreed on a common mapping from switch events to sets of histories. In this example, the mapping of a switch event of client $c$ with switch value $v$ is the set of all histories starting with invocation $\operatorname{propose}(v)$ from a client $c^{\prime} \neq c$ and containing only invocations from clients other than $c$. Hence, a client switching to Backup with switch value $v$ indicates to Backup that any history in the set mapped to $v$ is a possible linearization of Quorum's execution.

Quorum is speculatively linearizable. We prove that Quorum is speculatively linearizable in two steps. First, we show that Quorum satisfies the following three invariants. Then we show that those invariants imply speculative linearizability.

I1: If some client $c$ decides value $v$ then all clients that switch, either before or after $c$ decides, do so with value $v$.

I2: If some client $c$ decides value $v$ then all clients that decide do so with value $v$.

I3: All clients that switch or decide do so with a value that was proposed before they switch or decide.

In Quorum, if there are no faults nor contention, a client broadcasting a proposal will receive identical accept messages from all server processes and will return the value contained in the accept messages. However, if contention causes proposals from different clients to be received in different orders at different servers, or if a server fails or messages are lost, clients will switch to Backup because different accept messages will be received or not enough messages will be received before the timers expire.

Observe that if a client returns a value $v$ in Quorum then all servers will reply with $\operatorname{accept}(v)$ to all the other clients. This is because a client only returns value $v$ if all servers send it accept messages with value $v$, and a server always responds with the same accept message. Hence, if some client decided, all the other clients will either return the same value $v$ or will switch to Backup with the initialization value $v$ in case message loss or server crashes cause their timers to expire. From these observation we can conclude that the invariants I1 and I 2 are satisfied by any trace of Quorum.

Moreover, as servers respond with an accept message containing the first proposal received, client processes return or switch with a value that was proposed before. Hence, the invariant $\mathrm{I} 3$ holds.

We now prove that any trace satisfying I1, I2, and I3 satisfies speculative linearizability. Consider a trace $t$ of Quorum that satisfies I1, I2, and I3.

According to the definition of speculative linearizability for the first phase, we begin by showing that $t$ is linearizable. If no client decides then the trace $t$ is trivially linearizable. Therefore assume that some clients decide. The invariant I 2 implies that all decisions in the trace are the same. Let $v$ be the common decision. The invariant I3 implies that some client, noted winner, proposed $v$ before any client decided $v$. Let the history $h$ be such that $h$ starts with winner's proposal and the sub-sequence of $h$ starting at position 2 is equal to the subsequence of $t$ containing all the proposals of the clients that decide and that are not winner. The history $h$ represents a linearization of trace $t$. We satisfy our definition of linearizability by associating to each decision from a client $c$ the history $h$ truncated just after the proposal of $c$. The linearizability of Quorum follows.

To prove speculative linearizability, it remains to show that we can associate appropriate histories to each switch event in $t$. We consider two cases.

First, assume that some clients decided. Then, by invariant I1 we know that clients decide or switch with the same value $v$. Let history $h$ be as defined above. To each switch event in $t$ we associate $h$. By definition of $h, h$ starts with the common decision $v$ and all clients that switch do so with value $v$. Moreover the clients that switch do not decide; their invocations thus do not appear in $h$. Hence, the association from switch events to histories respects the common mapping. Moreover, $h$ is a linearization of trace $t$. Therefore, each history associated to a switch event is a linearization of $t$. Finally, the longest common prefix of all histories associated to switch events is obviously $h$ itself, and $h$ is a linearization of trace $t$. We conclude that $t$ satisfies speculative linearizability.

Now assume that no client decides. In this case the trace is trivially linearizable. Moreover, it is easy to associate histories to switch events, so that the longest common prefix of all such histories is empty. We thus trivially have that $t$ satisfies speculative linearizability.

Note that a client that does not fail returns or switches at the latest when its timer expires. Quorum is thus wait-free.

Backup is speculatively linearizable. As for Quorum, we proceed by first abstracting Backup using simple invariants and then proving that those invariants imply speculative linearizability. Since Paxos has been proved correct in the past [13], we can trivially abstract Backup using the following two invariants:

I4 All clients decide the same value.

I5 All clients decide a switch value previously submitted by some client.

Consider a trace $t$ satisfying invariants I4 and I5. We consider two cases. First assume that all switch values are the same and equal to $v$. Then, by definition of the common mapping from switch events to histories, for all associations of histories to switch events respecting the common mapping, the longest common prefix $h$ of the histories associated to switch values is such that $h$ starts with an invocation propose $(v)$ from a client $c$ that doesn't execute in $t$ and contains only invocations by clients that don't execute in $t$. Let $t_{h}$ be the trace represented by history $h$. By invariants I 4 and I5 we know that all clients decide value $v$ in $t$. Let $t^{\prime}$ be the trace $t$ where switch calls are replaced by the pending invocation they contain. Consider the concatenation $t_{h} @ t^{\prime}$ of $t_{h}$ and $t^{\prime}$. Let the history $h^{\prime}$ be the concatenation of $h$ and some ordering of the proposals in $t^{\prime}$. Because $h$ represents $t_{h}$ and because all clients decide $v$ in $t$, we have that $h^{\prime}$ is a linearization of $t_{h} @ t^{\prime}$. Hence, $t$ satisfies speculative linearizability.

Now suppose that at least two switch values are different. Then for any association of histories to switch events respecting the common mapping, the longest common prefix of the histories associated to switch events is the empty history. From invariants I4 and I5 we have that all processes decide on one of the switch values submitted before any decision. Hence, $t$ is linearizable, and so is the concatenation of the empty history and $t$. Therefore, $t$ satisfies speculative linearizability.

We have proved that Quorum and Backup satisfy speculative linearizability. Therefore, using the intra-object composition theorem that we will prove below, we conclude that the composition of Quorum and Backup is a linearizable implementation of consensus.

Using speculative linearizability, we have optimized the Paxos algorithm to obtain an algorithm that has a latency of two message delays in the absence of faults and contention. Implementing this optimization by modifying the Paxos algorithm would have meant modifying a notoriously intricate distributed algorithm. Instead, by using our framework, we were able to optimize Paxos without modifying it. Moreover, thanks to the intra-object composition theorem, we easily proved the optimized protocol correct just by proving the optimization speculatively linearizable and by relying on the correctness of Paxos. 


\subsection{Shared-Memory Consensus}

To illustrate the broad applicability of our framework we now apply it to an algorithm in the shared-memory model. We consider a consensus implementation in an asynchronous shared-memory system.

Consensus can be implemented on modern microprocessors using the wait-free compare-and-swap (CAS) instruction, but this instruction may be slower than an atomic register access. It has been proved [11] that wait-free consensus cannot be implemented with registers.

However, assuming that all executions are contention free (i.e. sequential), Figure 1 implements consensus using only registers. Hence the following question: is it possible to devise an object that uses only registers in contention-free executions but that always executes correctly? We obtain such an object by composing a registerbased speculation phase called RCons, shown in Figure 2, and a CAS-based speculation phase called CASCons, shown in Figure 3. The CAS-based speculation phase is a straightforward implementation of consensus using the CAS operation. Both speculation phase are inspired by [25]. It turns out that these speculation phases, like many other speculation-based objects, are instances of our framework.

The register-based consensus uses a wait-free splitter algorithm [19]. The splitter guarantees that at most one process returns with true and all others return with false. Moreover, it guarantees that, in the absence of contention, exactly one process returns with true. A splitter can be implemented using only registers, as in our example implementation in Figure 2.

A client invokes the register-based speculation phase using the function propose $(\mathrm{val})$, where $\mathrm{val}$ is the value that it proposes. To simplify the notation, we assume that, at the time of the call, the caller identifier is stored in the special variable $c$. The registerbased speculation phase, RCons, can return the content of register $V$, or it can switch to the CAS-based speculation phase, CASCons, by invoking the function switch-to-CASCons $(V)$ and returning its result. In the latter case, we say that a client switches with value $v$, where $v$ is the content of register $V$ when it was last read. Such switching is the basic mechanism of composing speculation phases in our framework.

As for Quorum and Backup, we will show that the composition of the phases RCons and CASCons form a linearizable implementation of consensus by a modular reasoning. Thanks to the intra-object composition theorem, we will get the correctness of the composition of RCons and CASCons by analyzing each in isolation.

Let us now show that the RCons and CASCons algorithms are speculatively linearizable.

As for Quorum and Backup, we will first consider RCons in isolation and, given an arbitrary execution of $R C$ Cons, we will show that we can associate appropriate histories to the responses returned and to the switch calls. We will then consider CASCons in isolation and show that, given an arbitrary execution of CASCons and an arbitrary association of histories to the switch calls of CASCons (respecting the common mapping), we can associate appropriate histories to the responses appearing in the trace.

We first show that the invariants I1, I2, and I3 defined for Quorum also hold of the traces of $R C$ Cons. To establish these invariants, we first observe the following: By property of the splitter, at most one client executes lines 15 to 18 . Moreover, if one client $c$ does so and returns, it makes sure that other clients will either return with its value at line 12 or switch with its value at line 27 . Indeed, if the variable Contention is false at line 16, it means that no process got past line 23. Therefore, all processes that switch will find $c$ 's value in variable $V$ at line 25 . We conclude that the invariants I1 and I2 hold on all executions of RCons:

\section{1: Object $R$ Cons}

2: //Shared Register $V$, Initially $\perp$; and $D$, Initially $\perp$

3: //Shared Register Contention $\in\{$ true, false $\}$, Initially false

4: //Shared Registers: $Y \in\{$ true, false $\}$, Initially false; and $X$

5: //Local Variable $v$; local constant $c$ denoting the caller's identifier

6: Function propose $(v a l)$ :

7: $v \leftarrow$ val

8: if $D \neq \perp$ then

9: return $D$

10: end if

11: if splitter ()$=$ true then

12: $\quad V \leftarrow v$

13: $\quad$ if $\neg$ Contention then

14: $\quad D \leftarrow v$

15: return $v$

16: else

17: return switch-to-CASCons $(v)$

18: end if

19: else

$$
\text { Contention }=\text { true }
$$

if $V \neq \perp$ then

$v \leftarrow V$

end if

return switch-to-CASCons $(v)$

end if

Function splitter():

27: $X \leftarrow c / /$ Remember that $c$ is the caller's identifier

28: if $Y=$ true then

30: end if

31: $Y \leftarrow$ true

32: if $X=c$ then

33: return true

34: else

35: return false

36: end if

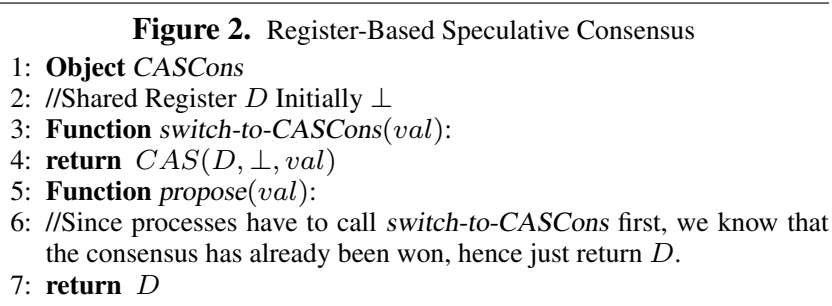

Figure 3. CAS-Based Speculative Consensus

I1: If some client $c$ returns value $v$ then all clients that switch, either before or after $c$ returns, do so with value $v$.

I2: If some client $c$ returns value $v$ then all clients that return do so with value $v$

Next, observe that all clients return or switch with their own value or with the content of register $V$, which can only contain proposed values. Consequently, the invariant $\mathrm{I} 3$ is also an invariant on all executions of RCons:

I3: All clients that switch do so with a value that was proposed before they switch.

Similarly, the CASCons phase can be abstracted by the same invariants that we used to abstract the Backup speculation phase:

I4 All clients return the same value.

I5 All clients return a switch value previously submitted by some client.

When we proved that Quorum and Backup are speculatively linearizable, we showed that the invariants I1, I2, and I3 imply speculative linearizability of the first phase and that the invariants 
I4 and I5 imply the speculative linearizability of the second phase. Since we established that the traces of RCons satisfy I1, I2, and I3 and that the traces of CASCons satisfy I4 and I5 we can conclude that RCons and CASCons satisfy speculative linearizability.

We will now formally define speculative linearizability.

\section{Trace Properties}

In this section we define trace properties and the operations they support. Trace properties are our model of distributed systems.

A trace property is a set of finite sequences of events. We use trace properties to describe the set of all finite sequences that a system can generate, as well as the desired properties of systems, such as linearizability with respect to a given abstract data type. We restrict ourselves to finite sequences because our work deals with safety properties only.

Sequences. We write $[1 . . n]$ for the set $\{1,2, \ldots, n\}$. A sequence $s$ of length $n$ with elements from a set $E$ is a function $s:[1 . . n] \rightarrow E$. We denote $n$ by $|s|$. We write $E^{*}$ for the set of all sequences of elements in $E$. We write $\left[e_{1}, e_{2}, \ldots, e_{n}\right]$ for the sequence $s$ such that $s(1)=e_{1}, s(2)=e_{2}, \ldots$, and $s(n)=e_{n}$. If $s=\left[e_{1}, e_{2}, \ldots, e_{n}\right]$ then $s:: e_{n+1}$ is the sequence $\left[e_{1}, e_{2}, \ldots, e_{n}, e_{n+1}\right]$. If $s=\left[e_{1}, e_{2}, \ldots, e_{n}\right]$ and $s^{\prime}=$ $\left[e_{1}^{\prime}, e_{2}^{\prime}, \ldots, e_{n}^{\prime}\right]$ then $s::: s^{\prime}=\left[e_{1}, \ldots, e_{n}, e_{1}^{\prime}, \ldots, e_{n}^{\prime}\right]$ and, if $m<$ $|s|$, we write $s_{\mid m}$ for the the sequence $\left[e_{1}, e_{2}, \ldots, e_{m}\right]$. We say that a sequence $s$ is a prefix of a sequence $s^{\prime}$ iff there exists a sequence $s^{\prime \prime}$ such that $s^{\prime}=s::: s^{\prime \prime}$. We say $s$ is a strict prefix of $s^{\prime}$ iff the $s^{\prime \prime}$ is non-empty. Given a set of sequences $P$, the longest common prefix of set $P$ is the longest among the sequences $s^{\prime}$ such that for all sequence $s \in P, s^{\prime}$ is a prefix of $s$.

Multisets. We represent multisets of elements of set $E$ by a multiplicity function $E \rightarrow \mathbb{N}$. Given two multisets $m_{1}$ and $m_{2}$ and $e \in E$, we define $\left(m_{1} \cup m_{2}\right)(e)=\max \left(m_{1}(e), m_{2}(e)\right)$ and $\left(m_{1} \uplus m_{2}\right)(e)=m_{1}(e)+m_{2}(e)$. Moreover $m_{1} \subseteq m_{2}$ iff for all $e \in E, m_{1}(e) \leq m_{2}(e)$. Let elems $: E^{*} \rightarrow(E \rightarrow \mathbb{N})$ be a function that given a sequence returns a multiset representing all the elements found in the sequence and their number of occurences. Given a sequence $s$ we write $e \in s$ iff elems $(s)(e)>0$.

Trace Properties. A trace is a sequence of actions which represents events happening at the interface between a system and its environment. An event occurs at some point in time and has no duration. We can approximate invoking a method and starting to execute it as one action. The execution of a method from start to finish, however, is typically not an action because it has a positive duration in time. We would represent it with an invocation action and a return action.

We classify actions into input and output actions. This classification is especially important to define composition of systems, because we need to know which actions of a component affect another component. The classification is described by a signature. A signature sig is a pair (in, out) where in is a set of input actions and out is a disjoint set of output actions. We require in and out to be disjoint. We denote by acts(sig) the set of all actions in signature sig. When all the actions of a trace $t$ belong to acts(sig) we say that $t$ is a trace in sig.

Definition 1. A trace property is a pair $P=$ (sig, Traces) where sig is a signature and Traces is a set of traces in acts(sig).

We denote sig by $\operatorname{sig}(P)$ and Traces by $\operatorname{Traces}(P)$. We allow to write $i n(P)$ instead of $i n(\operatorname{sig}(P))$ and similarly for the other sets of actions defined above.

We say that a trace property $Q$ satisfies a trace property $P$, denoted $Q \models P$, if $Q$ 's signature is equal to $P$ 's signature and the set of trace of $Q$ is a subset of the set of traces of $P$ :

$$
Q \models P \Leftrightarrow \quad(\operatorname{sig}(Q)=\operatorname{sig}(P) \wedge \operatorname{Traces}(Q) \subseteq \operatorname{Traces}(P)) .
$$

Composition of Trace Properties. Trace properties may be composed only if their signatures are compatible. Signatures $\operatorname{sig}_{1}$ and $\operatorname{sig}_{2}$ are compatible if and only if they have no output actions in common, that is out( $\left.\operatorname{sig}_{1}\right)$ is disjoint from out $\left(\operatorname{sig}_{2}\right)$. As a result, $\operatorname{out}\left(\operatorname{sig}_{1}\right) \cap \operatorname{acts}\left(\operatorname{sig}_{2}\right) \subseteq i n\left(\operatorname{sig}_{2}\right)$ and vice versa. We define the projection $\operatorname{proj}(t, A)$ of a trace $t$ onto a set $A$ of actions as the sequence obtained by removing all actions of $t$ that are not in $A$. For example, $\operatorname{proj}\left(\left[x, y, x^{\prime}, z, y^{\prime}, z, y, z, y\right],\left\{x^{\prime}, y^{\prime}\right\}\right)=\left[x^{\prime}, y^{\prime}\right]$.

Definition 2. If $\mathrm{sig}_{1}$ is compatible with $\mathrm{sig}_{2}$ then the composition $P=P_{1} \| P_{2}$ is such that:

- The signature $\operatorname{sig}(P)$ is such that in $(P)=\left(\right.$ in $\left.\left(P_{1}\right) \cup i n\left(P_{2}\right)\right) \backslash$ $\left(\operatorname{out}\left(P_{1}\right) \cup \operatorname{out}\left(P_{2}\right)\right)$ and out $(P)=\operatorname{out}\left(P_{1}\right) \cup \operatorname{out}\left(P_{2}\right)$.

- The set of traces Traces $(P)$ is the largest set of traces in acts $(P)$ such that if $t \in \operatorname{Traces}(P)$ then $\operatorname{proj}\left(t, \operatorname{acts}\left(P_{1}\right)\right) \in \operatorname{Traces}\left(P_{1}\right)$ and $\operatorname{proj}\left(t, \operatorname{acts}\left(P_{2}\right)\right) \in \operatorname{Traces}\left(P_{2}\right)$

Intuitively, composition requires components to execute at the same time the actions that appear as input of one component and output of the other. Actions that appear in a unique component are executed asynchronously from the other components. Composition has the following property:

Property 1. If $Q_{1} \models P_{1}$ and $Q_{2} \models P_{2}$ then $Q_{1}\left\|Q_{2} \mid=P_{1}\right\| P_{2}$.

Projection of Trace Properties.

Definition 3. Given a trace property $P$ we define its projection $\operatorname{proj}(P, A)$ onto a set of actions $A$ such that $\operatorname{sig}(\operatorname{proj}(P, A))=$ $(\operatorname{in}(P) \cap A, \operatorname{out}(P) \cap A)$ and $\operatorname{Traces}(\operatorname{proj}(P, A))=\left\{t \mid \exists t^{\prime} \in\right.$ $\left.\operatorname{Traces}(P) \cdot t=\operatorname{proj}\left(t^{\prime}, A\right)\right\}$

Applying the proj operator to a trace property $P$ amounts to removing all the actions not in $A$ from $P$ 's interface.

\section{Linearizability}

Our notion of speculative linearizability is itself based on a new definition of linearizability. This definition is interesting in its own right because it enables a more local form of reasoning than the original definition. Moreover, it allows for repeated events, which are the norm in practice. The technical report [9] contains a proof that our new definition of linearizability is equivalent to the classical definition.

\subsection{Abstract Data Types}

Abstract Data Types (ADTs) formalize our intuitive understanding of how sequential objects may behave.

In order to simplify our discussion in the rest of the paper, we deviate from the usual, state-machine based, definition of abstract data types. However we do so without loss of generality. Traditionally, the allowed behaviors are described using an ad-hoc state machine that given a state and an input transitions to a new state and produces an output. Instead, we determine outputs from the input history seen so far using an output function. Computation of the output function amounts to replaying the execution of the statemachine description.

Definition 4. An ADT $T$ is a tuple $T=\left(I_{T}, O_{T}, f_{T}\right)$ where $I_{T}$ is a non-empty set, whose members we call inputs, $O_{T}$ is a disjoint non-empty set whose members we call outputs, and $f_{T}: I_{T}^{*} \rightarrow O_{T}$ is an output function.

In the rest of the paper, we consider objects of some fixed ADT $T=\left(I_{T}, O_{T}, f_{T}\right)$.

Example 1. We consider a consensus object with operations of the form $\left(p(v), d\left(v^{\prime}\right)\right)$ with $v$ and $v^{\prime}$ belonging to some set $V_{\text {Cons. }}$ 
$p(v)$ is a shorthand for "propose $(v)$ " and $d(v)$ for "decide $(v)$ ". The consensus object must guarantee that a unique value $v$ may be decided and that at any point a decided value has been proposed before. Hence, because we are considering sequential executions, the first proposed value must be decided in all subsequent operations. Formally, we define the ADT Cons such that $I_{\text {Cons }}=\left\{p(v) \mid v \in V_{\text {Cons }}\right\}, O_{\text {Cons }}=\left\{d(v) \mid v \in V_{\text {Cons }}\right\}$, $f_{\text {Cons }}\left(\left[p\left(v_{1}\right), p\left(v_{2}\right), \ldots, p\left(v_{n}\right)\right]\right)=d\left(v_{1}\right)$

\subsection{A Trace Model of Concurrent Objects}

We consider a set $C$ of asynchronous sequential processes called clients. Clients use a concurrent object of ADT $T$ by calling the object's invocation function with one of the ADT's inputs, and the invocation function returns one of the ADT's outputs. We denote a sequence of interaction between clients and object as follows. Suppose that the following sequence of interactions between clients and object is observed: Client $c_{1}$ invokes input in ${ }_{1}$; Client $c_{2}$ invokes input $i_{2}$; Client $c_{2}$ returns with output out $t_{2}$; Finally client $c_{1}$ returns with output out . This sequence of interactions is denoted by the sequence of actions

$$
\left[\operatorname{inv}\left(c_{1}, \operatorname{in}_{1}\right), \operatorname{inv}\left(c_{2}, \operatorname{in}_{2}\right), \operatorname{res}\left(c_{2}, \text { in }_{2}, \text { out }_{2}\right), \operatorname{res}\left(c_{1}, \text { in }_{1}, \text { out }_{1}\right)\right]
$$

Signature of a Concurrent Object Formally, a sequence of interactions between a concurrent object and its clients is a trace of actions in the signature $\operatorname{sig}_{T}$, where the inputs $i n\left(s i g_{T}\right)$, called invocation actions, are of the form $\operatorname{inv}(c, n$, in) and the outputs $\operatorname{out}\left(\operatorname{sig}_{T}\right)$, called response actions, are of the form res $(c, n$, in, out $)$ for some client $c$, natural number $n$, in $\in I_{T}$, and out $\in O_{T}$.

The reader will notice that the second parameter of the actions is not present in our preceding example. It will be used in Subsection 5 to define the signature of a speculation phase.

In Section 4 we define the trace property $\operatorname{Lin}_{T}$ such that a concurrent object described by a trace property $O$ is linearizable w.r.t. $T$ if and only if $O \subseteq \operatorname{Lin}_{T}$.

Underscore Notation. Throughout the paper we will use the underscore symbol "." in mathematical expressions. Each occurrence of this symbol is to be replaced by a fixed fresh variable. For example, we might say an action $a$ is an invocation action if $a=\operatorname{inv}\left({ }_{-},,_{-}\right)$, or equivalently, that if $a=\operatorname{inv}(c, n, i n)$ for some $c, n$, and in, then $a$ is an invocation action.

\subsection{A New Definition of Classical Linearizability}

In this section we define a trace property called linearizability, and noted $\operatorname{Lin}_{T}$. Informally, a trace in $\operatorname{sig}_{T}$ is said to be linearizable iff its actions may be reordered to form a sequential trace that:

1. Respects the semantics of the ADT $T$.

2. Preserves the order of non-overlapping operations.

Property 1 implies that clients accessing a linearizable object cannot tell whether the object produces sequential or concurrent traces. This is a crucial property because it hides concurrency from the view of application developers. Property 2 makes linearizability a local property. In other words, a system composed of linearizable objects is itself linearizable. We refer the reader to [12] for an indepth discussion about linearizability.

Instead of using the definition above we propose a new definition of linearizability that will simplify our task when we define speculation phases. It directly establishes a relationship between the linearizable traces and the ADT, without the intermediary of sequential traces.

Note that some definitions of linearizability [12] assume more or less explicitly that all inputs submitted are unique. This seems unwarranted as many algorithms qualified as implementing a linearizable object are deemed correct without requiring this assump- tion. Our definition does not rely on this assumption and it coincides with the other definitions on traces satisfying the assumption.

Consider a trace $t$ in $\operatorname{sig}_{T}$.

Definition 5. Trace $t$ is linearizable iff it is well-formed and it admits a linearization function $g:[1 . .|t|] \rightarrow I_{T}^{*}$.

We define linearization functions and well-formed traces below.

To ensure that our definition corresponds to the usual one, in the technical report [9] we also formalize the usual definition of linearizability [12], by defining when a trace is linearizable* , even in case of repeated events. We then prove the equivalence between the two definitions.

Theorem 1. A trace in $\operatorname{sig}_{T}$ is linearizable if and only if it is linearizable*.

\subsection{Linearization Functions}

In the rest of the paper we call sequences of inputs histories. A linearization function maps the indices of $t$ to histories which must satisfy two properties:

First, applying the ADT's output function to the history associated with a response index must yield the output contained in the response. Hence a linearization function may be seen as giving an explanation of the outputs observed, in term of the ADT's semantics.

Second, the history associated to a response index must only contain inputs invoked before index $i$ and for all pairs of histories corresponding to response indices, one is a strict prefix of the other. This second condition gives us a total order on the response indices of $t$ (the prefix order on histories) which corresponds to the reordering needed in the original definition.

A history corresponding to some response index $i$ in a trace $t$ according to a linearization function is said to be a linearization of the (concurrent) trace $t_{\mid i}$.

We now state this definition formally:

Definition 6. Linearization Function. A function $g$ is a linearization function for $t$ iff

- Function g explains trace $t$.

- Function $g$ and trace $t$ satisfy the validity $(t, g)$ and commit$\operatorname{order}(t, g)$ predicates.

Definition 7. Explains. We say that function $g$ explains trace $t$ iff for all index $i \in[1 . .|t|]$, if $t(i)=\operatorname{res}\left({ }_{-},,_{-}\right.$, out $)$then out $=$ $f_{T}(g(i))$.

Consider a function $g:[1 . . n] \rightarrow I_{T}^{*}$ that explains trace $t$.

Definition 8. Commit Index and Commit Histories. If $i \in[1 . . n]$ is such that $t(i)=$ res $\left({ }_{-},,_{-},{ }_{-}\right)$then we say that $i$ is a commit index and that $g(i)$ is a $(t, g)$-commit history.

Definition 9. Sequence of Previous Inputs. We define the sequence of previous inputs at $i$ in $t$, inputs $(t, i)$, as the sequence of all inputs submitted before index $i$ in trace $t$. Formally, for all $i \in[1 . .|t|]$, $\mid$ inputs $(t, i)|=| \operatorname{proj}\left(t_{\mid i}, \operatorname{in}\left(\operatorname{sig}_{T}\right) \mid\right.$, and for all $j \in[1 . . \mid$ inputs $(t, i) \mid], \operatorname{proj}\left(t_{\mid i}, \operatorname{in}\left(\operatorname{sig}_{T}\right)\right)(j)=$ $\operatorname{inv}\left({ }_{-},-\right.$, inputs $\left.(t, i)(j)\right)$.

Definition 10. Valid Commit Index For all index $i \in[1 . .|t|]$ such that $t(i)=\operatorname{res}\left({ }_{-},\right.$, in,,$)$, we say that $i$ is $(t, g)$-valid iff elems $(g(i)) \subseteq$ elems(inputs $(t, i))$ and the input in is the last element of the history $g(i)$.

In other words, if $t(i)=\operatorname{res}\left({ }_{-},\right.$, in,,-$)$then $g(i)$ is a permutation of the sequence of previous inputs at $i$ and ends with the input in.

We now define validity and commit-order:

Definition 11. Validity $(t, g)$. All commit indices in $t$ are $(t, g)$ valid. 
Definition 12. Commit Order $(t, g)$ : For all pairs of distinct commit indices $(i, j)$, either $g(i)$ is a strict prefix of $g(j)$ or $g(j)$ is a strict prefix of $g(i)$.

Example 2. Consider the following trace $t$ :

$\left[\operatorname{inv}\left(c, i n_{1}\right), \operatorname{inv}\left(c^{\prime}, \operatorname{in}_{2}\right), \operatorname{res}\left(c^{\prime}, \operatorname{in}_{2}, f_{T}\left(\left[i_{2}\right]\right)\right), \operatorname{res}\left(c, i_{1}, f_{T}\left(\left[i_{2}, i n_{1}\right]\right)\right)\right]$. Consider a function $g$ such that $g(3)=\left[\mathrm{in}_{2}\right]$ and $g(4)=\left[\mathrm{in}_{2}, \mathrm{in}_{1}\right]$. The function $g$ explains $t$. Moreover $t$ and $g$ satisfy validity and commit-order. Hence $g$ is a linearization function for $t$.

\subsection{Well-Formed Traces}

The well-formedness of traces is a basic requirement. For example, it states that a response should be given to a client only if it previously issued an invocation.

Given a client $c$, let $A c t_{T}(c)$ be the union of the sets $\left\{\operatorname{inv}\left(c,_{-}\right.\right.$, in $) \mid$in $\left.\in I_{T}\right\}$ and $\left\{\operatorname{res}\left(c,_{-}\right.\right.$, in, out $) \mid$in $\in I_{T} \wedge$ out $\left.\in O_{T}\right\}$.

Definition 13. Client sub-trace. The client sub-trace sub $(t, c)$ of trace $t$ in $\operatorname{sig}_{T}$ is the trace $s u b(t, c)=\operatorname{proj}\left(t, A c t_{T}(c)\right)$.

Definition 14. Well-formed client sub-trace. A client sub-trace $t$ is well-formed iff $t(1)$ is such that $t(1)=\operatorname{inv}\left({ }_{-},,_{-}\right)$and for all $i \in[1 . .|t|-1]$, if $t(i)=\operatorname{inv}\left({ }_{-},-\right.$, in $)$, then $t(i+1)=\operatorname{res}\left({ }_{-},-\right.$, in,,$\left.{ }_{-}\right)$.

Definition 15. Well-formed traces. We say that trace $t$ in sig $_{T}$ is well-formed if and only if all its client sub-traces are well-formed.

Note that invocations with no corresponding response may appear in a well-formed trace. We say that those invocations are pending.

\subsection{The $\operatorname{Lin}_{T}$ Trace Property}

We define the trace property $\operatorname{Lin}_{T}$ such that the signature $\operatorname{sig}\left(\operatorname{Lin}_{T}\right)$ is $\operatorname{sig}_{T}$ and the set $\operatorname{Traces}\left(\operatorname{Lin}_{T}\right)$ is the set of all traces satisfying linearizability.

Finally, consider a system $S$ whose behavior is denoted by a trace property $P$. We say that the system $S$ implements the ADT $T$ if and only if $\operatorname{proj}\left(S, \operatorname{sig}_{T}\right) \models \operatorname{Lin}_{T}$.

\section{Speculative Linearizability}

We next present our main results: a precise definition of speculative linearizability, and the proof that a composition of implementations that are speculatively linearizable implementations is itself speculatively linearizable. This section presents a trace-based formalization. We summarize an alternative, automata-based, formalization in Section 6.

\subsection{Speculation Phases}

We now consider concurrent objects implemented by several speculation phases. Clients use a speculation phase by calling the object's invocation functions and the invocation functions return outputs to the clients. However, a speculation phase also accepts initialization calls from clients and it may switch to another speculation phase.

Moreover, each speculation phases is identified by a natural number and a speculation phase identified by $n$ may only switch to a speculation phase identified by $n+1$. Clients start by accessing speculation phase 1 and continue to do so as long as they return in the phase 1 . However, speculation phase 1 may switch to phase 2 . In this case it passes an initialization value and its pending input to phase 2 . A client which returns in phase 2 stops accessing speculation phase 1 and instead uses phase 2 for its next invocations.

Let $S_{1}$ and $S_{2}$ be two consecutive speculation phases. Suppose that client $c_{1}$ invokes input $i_{1}$ on $S_{1}$; client $c_{2}$ invokes input in ${ }_{2}$ on $S_{1}$; client $c_{2}$ switches from $S_{1}$ to $S_{2}$ providing initialization value $v$; client $c_{1}$ returns response out $t_{1}$ from $S_{1}$; finally client $c_{2}$ returns response out 2 from $S_{2}$. This sequence of interactions is denoted by this sequence of events, from now on called actions:

$$
\begin{aligned}
& {\left[\operatorname{inv}\left(c_{1}, 1, \operatorname{in}_{1}\right), \operatorname{inv}\left(c_{2}, 1, \operatorname{in}_{2}\right), \operatorname{swi}\left(c_{2}, 2, \operatorname{in}_{2}, v\right),\right.} \\
& \left.\operatorname{res}\left(c_{1}, 1, \operatorname{in}_{1}, \text { out }_{1}\right), \operatorname{res}\left(c_{2}, 2, \operatorname{in}_{2}, \text { out }_{2}\right)\right]
\end{aligned}
$$

Instead of formalizing the signature of a single speculation phase identified by a natural number we generalize to the signature of a composition of speculation phases. We now assume that a speculation phase may be composed of several other speculation phases.

Let $\mathbb{N}_{<}^{2}=\left\{(m, n) \mid(m, n) \in \mathbb{N}^{2} \wedge m<n\right\}$. We denote a speculation phase by an element of $\mathbb{N}_{<}^{2}$. A speculation phase $(m, n) \in \mathbb{N}_{<}^{2}$ describes the possible behaviors of the composition of speculation phases from $m$ to $n$.

We now explain why we make this generalization.

We would like to define a property $P(n)$ of speculation phase $n$, for $n \in \mathbb{N}$, such that for all $m \in \mathbb{N}$ the composition $P(1)\|P(2)\| \ldots \| P(m)$ implements $\operatorname{Lin}_{T}$. To prove that

$$
P(1)\|P(2)\| \ldots \| P(m) \models \operatorname{Lin}_{T}
$$

we generalize $P(n)$ to $P(m, n)$ in order to use induction.

We would like $P(m, n)$ to be such that:

$$
\begin{aligned}
& \text { For all natural number } n>1, P(1, n)=\operatorname{Lin}_{T} \\
& \text { For all }(m, n) \in \mathbb{N}_{<}^{2} \text { and }(n, o) \in \mathbb{N}_{<}^{2}, \\
& \qquad P(m, n) \| P(n, o) \models P(m, o)
\end{aligned}
$$

Using induction and equation 2 we have that for all $n>1$,

$$
P(1,2)\|P(2,3)\| \ldots \| P(n-1, n) \models P(1, n) .
$$

Thus, with equation 1 , we have that for all $n>1$,

$$
P(1,2)\|P(2,3)\| \ldots \| P(n-1, n) \models \operatorname{Lin}_{T}
$$

Now suppose that we have a family of trace properties $Q_{1}, Q_{2}, \ldots, Q_{n}$, representing implementations of speculation phases, such that $Q_{i} \models P(i, i+1)$ for all $i \in[1 . . n]$. Let Comp $=Q_{1}\left\|Q_{2}\right\| \ldots \| Q_{n}$. By property 1 and equation 3, we have that $C o m p \models \operatorname{Lin}_{T}$.

Signature of a Speculation Phase We now define a signature $\operatorname{sig}_{T}(m, n$, Init $)$ which models the interface of a speculation phase $(m, n)$ that uses initialization values from the set Init. For all $m \in \mathbb{N}$ and any arbitrary non-empty set Init of initialization values, we define the set of switch actions as formed of all actions of the form swi $(c, m$, in, $v)$ where $c$ is a client, $m \in \mathbb{N}$, in $\in I_{T}$, and $v \in$ Init.

Definition 16. Signature of a speculation phase. For all $(m, n) \in$ $\mathbb{N}_{<}^{2}$ and any non-empty set Init of initialization values we define the signature $\operatorname{sig}_{T}(m, n$, Init) as the union of the sets of all invocation actions inv $\left({ }_{-}, O,_{-}\right)$, of all response actions res $\left({ }_{-}, o_{,},,_{-}\right)$, and of all switch actions swi(, $\left., o_{-},{ }_{-}\right)$, where $o \in[m . . n]$.

\subsection{Specification of a Speculation Phase}

In this section we propose a specification for speculation phases. We consider a set of initialization values Init, and a relation $r_{\text {init }} \in$ Init $\times I_{T}^{*}$ such that $r_{\text {init }}^{-1}$ is a total onto function. The relation $r_{\text {init }}$ associates a set of input sequences to any value in Init. Remember that the init values convey information about the execution of one phase to the next phase. We think of this information in terms of input histories which represent possible linearizations of the concurrent trace of a phase. We say the set of input sequences associated by $r_{\text {init }}$ with a value is the value's possible interpretations.

We will now define a trace property $\operatorname{SLin}_{T}(m, n)$, such that

- For all natural number $m, \operatorname{proj}\left(\operatorname{SLin}_{T}(1, m), \operatorname{acts}\left(\operatorname{sig}_{T}\right)\right) \models$ $\operatorname{Lin}_{T}$. 
- For all $(m, n) \in \mathbb{N}_{<}^{2}$ and $(n, o) \in \mathbb{N}_{<}^{2}$, $\operatorname{SLin}_{T}(m, n) \| \operatorname{SLin}_{T}(n, o) \models S \operatorname{Lin}_{T}(m, o)$.

We define the property $\operatorname{SLin}_{T}(m, n)$ in the same vein as $\operatorname{Lin}_{T}$. Consider a trace $t$ in $\operatorname{sig}_{T}(m, n$, Init).

Definition 17. Interpretation of Init Actions. We say that a function $f:[1 . .|t|] \rightarrow I^{*}$ is an interpretation of the init actions of $t$ iff for all index $i \in[1 . .|t|]$, if $t(i)=\operatorname{swi}\left({ }_{-}, m,{ }_{-}, v\right)$ then $(v, f(i)) \in r_{\text {init. }}$

Definition 18. Interpretation of Abort Actions. We say that a function $f:[1 . .|t|] \rightarrow I^{*}$ is an interpretation of the abort actions of $t$ iff for all index $i \in[1 . .|t|]$, if $\left.t(i)=\operatorname{swi}_{-}, n,,_{-}, v\right)$ then $(v, f(i)) \in r_{\text {init. }}$

Definition 19. Speculatively Linearizable. We say that trace $t$ is $(m, n)$-speculatively linearizable iff trace $t$ is $(m, n)$-well-formed and for all interpretation $f_{\text {init }}$ of the init actions of $t$, there exists two functions $g:[1 . .|t|] \rightarrow I_{T}^{*}$ and $f_{\text {abort }}:[1 . .|t|] \rightarrow I_{T}^{*}$ such that:

- $f_{\text {abort }}$ is an interpretation of the abort actions of $t$.

- $g$ is an $\left(f_{\text {init }}, f_{\text {abort }}, m, n\right)$-speculative linearization function for $t$.

\subsection{Speculative Linearization Functions}

Intuition Behind the Definitions. Consider a speculation phase $S_{1}$ whose clients switch to speculation phase $S_{2}$. We require clients to switch with a value whose set of possible interpretations includes a possible linearization of the concurrent trace at the point where the client switches. Note that every history (1) of which all commit histories are prefix and (2) that contains only values that have been invoked is a possible linearization. This is the intuition behind the definition of Abort-Order property (Definition 32).

To ensure that phase $S_{2}$ continues executing consistently with what phase $S_{1}$ did, we require all its commit histories to have as a prefix one of the possible linearizations of $S_{1}$ 's trace. This is the intuition behind the Init Order property (Definition 31). However $S_{2}$ receives switch values that represent sets of histories, and cannot determine which history in this set is a possible linearization. Hence the quantifier alternation in Definition 19: for all possible interpretations of the init values, phase $S_{2}$ needs to ensure the existence of a proper linearization function.

Definition 20. Speculative linearization function. A function $g$ : $[1 . .|t|] \rightarrow I_{T}^{*}$ is a $\left(f_{\text {init }}, f_{\text {abort }}, m, n\right)$-speculative linearization function for $t$ iff:

- The function g explains trace $t$.

- The predicates Validity $\left(t, g, f_{\text {init }}, f_{\text {abort }}\right)$, Commit-Order $(t, g)$, Init-Order $\left(t, g, f_{\text {init }}, f_{\text {abort }}\right)$, and Abort-Order $\left(t, g, f_{\text {abort }}\right)$ hold.

Definition 21. Explains. We say that the function g explains trace $t$ iff for all index $i \in[1 . .|t|]$, if $t(i)=\operatorname{res}\left({ }_{-},{ }_{-},{ }_{-}\right.$out $)$then out $=$ $f_{T}(g(i))$

Consider a function $g:[1 . .|t|] \rightarrow I_{T}^{*}$ that explains trace $t$ and consider two functions $f_{\text {init }} \subseteq r_{\text {init }}$ and $f_{\text {abort }} \subseteq r_{\text {init }}$. In order to define validity, commit-order, init-order, and abort-order we need the definitions 22 to 28 below.

Definition 22. Commit Indices and Commit Histories. If $i \in$ $[1 . . n]$ is such that $t(i)=\operatorname{res}\left({ }_{-},,_{-},{ }_{-}\right)$then we say that $i$ is a $(t, m, n)$-commit index and that $g(i)$ is $a(t, g)$-commit history.

Definition 23. Init Indices and Init Histories. If $i \in[1 . . n]$ is such that $t(i)=\operatorname{swi}\left({ }_{-}, m,{ }_{-}, v\right)$ we say that $i$ is a $(t, m, n)$-init index and that $f_{\text {init }}(i)$ is $a\left(t, m, n, f_{\text {init }}\right)$-init history.

Definition 24. Abort Indices and Abort Histories. If $i \in[1 . . n]$ is such that $t(i)=\operatorname{swi}\left(-, n,{ }_{-}, v\right)$ we say that $i$ is $a(t, m, n)$-abort index and that $f_{\text {abort }}(i)$ is a $\left(t, m, n, f_{\text {abort }}\right)$-abort history.
The multiset $i v i\left(m, t, f_{\text {init }}, i\right)$ contains all the inputs known to to have been invoked in phases $(k, l)$, where $l \leq m$ :

Definition 25. Initially Valid Inputs.

$$
\begin{aligned}
& i v i\left(m, t, f_{\text {init }}, i\right)= \\
& \bigcup\left\{\text { elems }\left(f_{\text {init }}(v)\right) \cup\{\text { in }\} \mid \exists j<i . t(i)=\operatorname{swi}(-, m, \text { in }, v)\right\} .
\end{aligned}
$$

Note that, in the previous definition, $U$ is the multiset union (defined in Section 3) and corresponds to pointwise maximum of representation functions.

Now recall that inputs $(t, i)$ is the sequence of inputs that were invoked before $i$ in $t$.

Definition 26. Valid Inputs. We define

$$
v i\left(m, t, f_{\text {init }}, i\right)=i v i\left(m, t, f_{\text {init }}, i\right) \uplus \operatorname{elems}(\text { inputs }(t, i))
$$

Definition 27. Valid Commit Index. For all index $i$ such that $t(i)=\operatorname{res}\left({ }_{-},{ }_{-}\right.$, in,-$)$, we say that $i$ is $\left(t, g, f_{\text {init }}\right)$-valid if and only if elems $(g(i)) \subseteq v i\left(m, t, f_{\text {init }}, i\right)$ and the input in is the last element of history $g(i)$.

Definition 28. Valid Abort Index. For all index $i$ such that $t(i)=$ swi( $(, n, i n, v)$, we say that $i$ is $\left(t, f_{\text {init }}, f_{\text {abort }}\right)$-valid if and only if elems $\left(f_{\text {abort }}(v)\right) \cup\{$ in $\} \subseteq v i\left(m, t, f_{\text {init }}, i\right)$.

We now define validity, commit-order, init-order, and abortorder:

Definition 29. $\operatorname{Validity}\left(t, g, f_{\text {init }}, f_{\text {abort }}\right): A l l(t, m, n)$-commit indices are $\left(t, g, f_{\text {init }}\right)$-valid and all $(t, m, n)$-abort indices are $\left(t, f_{\text {init }}, f_{\text {abort }}\right)$-valid.

Definition 30. Commit $\operatorname{Order}(t, g)$ : For all pair of distinct commit indices $i, j$, either $g(j)$ is a strict prefix of $g(i)$ or $g(i)$ is a strict prefix of $g(j)$.

Definition 31. Init $\operatorname{Order}\left(t, g, f_{\text {init }}, f_{\text {abort }}\right)$ : The longest common prefix of all $\left(t, m, n, f_{\text {init }}\right)$-init histories is a strict prefix of all $(t, g)$ commit histories and of all $\left(t, m, n, f_{\text {abort }}\right)$-abort histories.

We assume that the longest common prefix of an empty set of histories is the empty history.

Definition 32. Abort $\operatorname{Order}\left(t, g, f_{\text {abort }}\right)$ : All $(t, g)$-commit histories are prefix of all $\left(t, m, n, f_{\text {abort }}\right)$-abort histories.

Note that if $m=1$, then $t$ has no init histories. Hence Init Order does not constrain commit and abort histories.

\subsection{Well-Formed Traces}

The well-formedness condition defines basic requirements on traces of a concurrent object.

Given a client $c$ let $\operatorname{Act}_{T}(c, m, n)$ be the set of actions such that

$$
\begin{aligned}
& \operatorname{Act}_{T}(c, m, n)=\left\{\operatorname{inv}(c, o, \text { in }) \mid o \in[m . . n] \wedge \text { in } \in I_{T}\right\} \\
& \quad\left\{\operatorname{res}(c, o, \text { in, } r) \mid o \in[m . . n] \wedge \text { in } \in I_{T} \wedge r \in O_{T}\right\} \\
& \quad \cup\left\{\operatorname{swi}(c, o, \text { in, } v) \mid o \in\{m, n\} \wedge \text { in } \in I_{T} \wedge r \in O_{T} \wedge v \in \text { Init }\right\}
\end{aligned}
$$

Note that the switch actions whose second parameter is not $m$ or $n$ are projected away.

Definition 33. Client sub-trace. The $(m, n)$-client-sub-trace of the trace $t$ is the trace $\operatorname{sub}(t, m, n, c)=\operatorname{proj}\left(t, A_{c t}(c, m, n)\right)$.

Definition 34. Well-formed client sub-trace. A ( $m, n)$-client subtrace $t_{c}$ is well-formed iff it is empty or:

- For all $i \in\left[1 . .\left|t_{c}\right|-1\right]$, if $t(i)=\operatorname{inv}\left({ }_{-},,_{-}\right.$, in $)$or $t(i)=$ swi $\left({ }_{-}, m\right.$, in,,$\left._{-}\right)$, then $t(i+1)=\operatorname{res}\left({ }_{-},{ }_{-}, i n,{ }_{-}\right)$or $t(i+1)=$ swi(,,$n$, in,,$)$.

- If $t_{c}$ contains an abort action then it is the last element of $t_{c}$.

- If $m \neq 1$ then $t_{c}(1)$ is an init action and there are no other init actions in $t_{c}$.

- If $m=1$ then $t_{c}(1)$ is an invocation and there are no init actions in $t_{c}$. 
Definition 35. Well-formed traces. We say that the trace $t$ is $(m, n)$-well-formed if and only if all its $(m, n)$-client sub-traces are well-formed.

\subsection{The $\operatorname{SLin}_{T}$ Trace Property}

We define the trace property $\operatorname{SLin}_{T}(m, n)$ as follows:

Definition 36. The $\operatorname{SLin}_{T}(m, n)$ is such that the signature $\operatorname{sig}\left(\operatorname{SLin}_{T}(m, n)\right) \quad$ is $\operatorname{sig}_{T}(m, n$, Init $) \quad$ and the set Traces $\left(\operatorname{SLin}_{T}(m, n)\right)$ is the set of all traces satisfying $(m, n)$ speculative linearizability.

Finally, consider a system $S$ whose behavior is denoted by a trace property $P$. We say that the system $S$ is a $(m, n)$-speculation phase if and only if $\operatorname{proj}(P, \operatorname{sig}(m, n)) \models A \operatorname{Lin}(m, n)$.

Theorem 2. For all natural number $m$, any initialization set Init, and any initialization relation $r_{\text {init, }}$

$$
\operatorname{proj}\left(\operatorname{SLin}_{T}(1, m), \operatorname{acts}\left(\operatorname{sig}_{T}\right)\right)=\operatorname{Lin}_{T}
$$

Proof. Compared to linearizability, speculative linearizability only adds constraints on the actions that are not in the signature $\operatorname{sig}_{T}$.

\subsection{Intra-Object Composition Theorem}

Our main theorem states that the composition of two speculation phases is also a speculation phase.

Theorem 3. Suppose that $S_{1} \models \operatorname{SLin}_{T}(m, n)$ and $S_{2} \models$ $\operatorname{SLin}_{T}(n, o)$, then $\operatorname{proj}\left(S_{1} \| S_{2}, \operatorname{sig}_{T}(m, o, \operatorname{Init})\right) \models \operatorname{SLin}_{T}(m, o)$.

The proof of this result in the technical report [9]. It consists of two pages in the current format. Among the key steps is the construction of a speculative linearization function $g$ that explains the traces of the composition of two phases. The function is constructed by merging two speculative linearization functions. To show that it is indeed a speculative linearization function we use a form of transitive reasoning to relate commit histories between different phases through abort histories. Among the properties of speculative linearizability, Validity is the most difficult to show, in part because it is sensitive to the fact that we allow duplicate events in our traces (something that even most existing developments of classic linearizability ignore).

\section{Automata Specification of Speculative Linearizability and Isabelle/HOL Proof}

We have also formalized in Isabelle/HOL an executable version of the specification of speculative linearizability. Our formalization is based on the theory of I/O-automata [21], which is formalized in Isabelle's IOA framework [22]. Our specification automaton corresponds to speculative linearizability instantiated for State Machine Replication [16] protocols (abbreviated SMR protocols). We wrote a mechanically-checked proof of the intra-object composition theorem for the automata specification. Thanks to this proof, it is now possible to obtain mechanically-checked proofs of speculative SMR protocols from the mechanically-checked proofs of its speculation phases taken in isolation. Moreover, our proof of the intra-object composition theorem shows that refinement proofs in our framework are practical.

The speculative approach to SMR protocols has been shown to yield some of the most efficient SMR protocols in practice [8]. Therefore, our formalization and the proof of the intra-object composition theorem enable for the first time scalable mechanicallychecked proofs of practical SMR protocols. A proof document and the corresponding Isabelle theories are available at the Archive of Formal Proofs [10].
Informal Description of the Automata Specification. The automata specification is a formalization of speculative linearizability for an ADT that we call the universal ADT. The output function of the universal ADT is the identity function. In other words, this ADT responds to an invocation with its full trace, in the form of a history. The universal ADT can be used as an abstraction for generic SMR protocols [8] because, given a linearizable implementation, it suffices to apply the output function of another ADT A to the responses in order obtain an implementation of A. We formalize the case where histories of inputs are used as initialization values and where the relation $r_{\text {init }}$ maps a history $h$ to the singleton set $\{h\}$. The specification automaton can be seen as an implementation of speculative linearizability in which all clients reside on a unique, centralized, process.

The automaton receives invocations and switch calls as inputs and produces responses and switch call outputs.

The specification automaton maintains a state with the following components:

- A history hist, representing the longest linearization made visible to a client (i.e. the longest commit history);

- for each client $c$, a phase phase $(c) \in\{$ Sleep, Pending, Ready, or Aborted \};

- for each client $c$, pending $(c)$, the last input submitted by $c$;

- a set InitHists containing the init histories received;

- two booleans: aborted and initialized.

We say that an input is pending if it is the last submitted input of a client $c$ whose phase is "Pending" and if it is not present in hist.

The automaton starts in a state where hist is the empty history, InitHists is the empty set, aborted and initialized are set to false, and for all clients $c$, phase $(c)=$ "Sleep".

We now describe how the automaton reacts to the inputs it receives, namely invocations and switch calls from the previous phase. When receiving a switch call from the previous phase by client $c$, if phase $(c)$ equals "Sleep" then the automaton adds the provided init history to the set InitHists and sets pending $(c)$ to the provided input. When receiving a new invocation by client $c$, if phase $(c)$ equals "Ready", the automaton sets pending $(c)$ to the input received. In both cases phase $(c)$ is then set to "Pending". This denotes the fact that client $c$ is waiting for a response to input pending $(c)$.

Moreover, the automaton nondeterministically performs one of the following actions:

A1 If initialized $=$ false and if there is at least one client $c$ such that phase $(c)$ is not "Sleep", then the automaton computes the longest common prefix of the histories in InitHists, assigns it to hist, and sets initialized to true.

A2 It selects a pending input, say from client $c$, appends it to hist, emits an output for client $c$ containing the new value of hist as response, and sets phase $(c)$ to "Ready".

A3 It sets aborted to true.

A4 If aborted $=$ true then a client $c$ such that phase $(c)$ is not "Aborted" is selected is phase $(c)$ is set to "Aborted". Moreover, the automaton emits a switch action containing an abort value $h^{\prime}$ such that hist is a prefix of $h^{\prime}$ and the inputs of $h^{\prime}$ which are not in hist are pending.

The precise definition of our specification automaton is available in the ALM entry [10] of the Archive of Formal Proofs.

Relation to the Trace Based Specification. The trace-based specification requires associating histories to responses (commit histories) and switch actions (init and abort histories). Because our ADT's output function is the identity and because $r_{\text {init }}(h)=\{h\}$, 
we have no choice but to associate to a response or switch action the very history it contains (remember that outputs and switch values are histories in this section). Hence commit histories are obtained by truncating hist at a pending request. Since hist grows by appending to it, we have that Commit Order is satisfied. Moreover, abort histories are obtained by appending some pending requests to hist, and at this point hist does not grow anymore. Hence Abort Order holds. Since hist is initialized with the longest common prefix of the init histories seen, Init Order also holds. Finally, hist grows by appending pending requests to it, Validity is also satisfied.

To gain more insight into speculative linearizability, one may also observe that any extension of history hist with some pending requests is a linearization of the current trace. Thanks to this observation, step A2 may be interpreted as selecting a possible linearization and producing an output that realizes it. Step A4 can be seen as passing one of the possible linearizations to the next speculation phase. Finally, in step A1, the automaton computes the weakest (given the init histories it received) under-approximation of the set of possible linearizations of the previous phase and selects one of them to initialize its execution.

Proof of the Intra-Object Composition Theorem. To prove the intra-object composition theorem in this formulation, we construct a refinement mapping [20] between the composition of two speculation phases and a single speculation phase. We prove the refinement mapping correct with the help of 15 state invariants about the composed automaton. Using the meta-theorem about refinement mappings (included in the IOA theory packaged with Isabelle/HOL), we conclude that the set of traces of the composition of two speculation phases is included in the set of traces of a single speculation phase. The proof is written in the structured proof language Isar [26] and consists of roughly of 500 proof steps. With the specification, it forms a total of 1600 lines of Isabelle/HOL code.

Our automata specification can be used as the basis for mechanically-checked refinement proofs of distributed protocols. Our proof of the composition is a good example of such a refinement proof and shows the practicality of the approach.

\section{Concluding Remarks}

We have presented speculative linearizability, an extension of the theory of linearizability. Our extension allows us to implement linearizable objects by composing independently devised speculation phases that are optimized for different situations. This form of intra-object composition complements the classical concept of inter-object composition, inherent to linearizability in its traditional form. We propose a formalized framework that enables, for the first time, scalable mechanically-checked development of linearizable objects of arbitrary abstract data types.

Our work can be viewed as generalization of [1] and [2]. The former work focused on contention-free executions and did not address composition: a protocol that aborts simply stops executing with no possibility of switching. In the latter work, a classification of the trace properties that are switchable is proposed. A global synchronization is required for switching. In contrast, our notion of speculative linearizability does not require the switching protocols to solve agreement and is, in that sense, much more general. Our work can also be viewed as a formalization of a generalized form of Abortable State Machine Replication (Abstract), an abstraction presented in an intuitive form in [8] to allow the construction of speculative Byzantine Fault Tolerant SMR protocols by composing independently designed speculation phases. Compared to [8], our work concerns arbitrary abstract data types, including one-shot ones, and generalizes the idea to linearizability. Being strictly more general, our framework can be used to reason about the faulttolerant algorithms developed following those approaches $[1,2,8]$.

\section{References}

[1] M. K. Aguilera, S. Frolund, V. Hadzilacos, S. L. Horn, and S. Toueg. Abortable and query-abortable objects and their efficient implementation. In PODC, 2007.

[2] M. Bickford, C. Kreitz, R. v. Renesse, and X. Liu. Proving hybrid protocols correct. In TPHOLs '01, 2001.

[3] W. J. Bolosky, D. Bradshaw, R. B. Haagens, N. P. Kusters, and P. Li. Paxos replicated state machines as the basis of a high-performance data store. In Proc. NSDI. USENIX Assoc., 2011.

[4] M. Burrows. The Chubby lock service for loosely-coupled distributed systems. In Proc. OSDI. USENIX Assoc., 2006.

[5] M. Castro and B. Liskov. A correctness proof for a practical byzantinefault-tolerant replication algorithm. Technical report, MIT, 1999.

[6] M. Castro and B. Liskov. Practical Byzantine fault tolerance. In OSDI, 1999.

[7] E. Gafni and L. Lamport. Disk paxos. Distributed Computing, 16(1):1-20, 2003.

[8] R. Guerraoui, N. Knezevic, V. Quema, and M. Vukolic. The Next 700 BFT Protocols. In EUROSYS, 2010.

[9] R. Guerraoui, V. Kuncak, and G. Losa. Speculative Linearizability. Technical Report 170038, EPFL, 2011.

[10] R. Guerraoui, V. Kuncak, and G. Losa. Abortable linearizable modules. In G. Klein, T. Nipkow, and L. Paulson, editors, The Archive of Formal Proofs. http://afp.sf.net/entries/ Abortable_Linearizable_Modules.shtml, March 2012. Formal proof development.

[11] M. Herlihy. Wait-free synchronization. ACM Trans. Program. Lang. Syst., 13:124-149, January 1991.

[12] M. P. Herlihy and J. M. Wing. Linearizability: a correctness condition for concurrent objects. ACM Trans. Program. Lang. Syst., 12(3):463$492,1990$.

[13] M. Jaskelioff and S. Merz. Proving the correctness of Disk Paxos. In G. Klein, T. Nipkow, and L. Paulson, editors, The Archive of Formal Proofs. http://afp.sf.net/entries/DiskPaxos.shtml, June 2005. Formal proof development.

[14] P. Jayanti. Adaptive and efficient abortable mutual exclusion. In PODC, 2003.

[15] R. Kotla, L. Alvisi, M. Dahlin, A. Clement, and E. Wong. Zyzzyva: speculative Byzantine fault tolerance. In SOSP, 2007.

[16] L. Lamport. The implementation of reliable distributed multiprocess systems. Computer Networks, 2:95-114, 1978.

[17] L. Lamport. On interprocess communication. part I: Basic formalism. Distributed Computing, 1(2):77-85, 1986.

[18] L. Lamport. On interprocess communication. part II: Algorithms. Distributed Computing, 1(2):86-101, 1986.

[19] L. Lamport. A fast mutual exclusion algorithm. ACM Trans. Comput. Syst., 5(1):1-11, 1987.

[20] N. Lynch and F. Vaandrager. Forward and backward simulations I: untimed systems. Inf. Comput., 121:214-233, September 1995.

[21] N. A. Lynch and M. R. Tuttle. An introduction to input/output automata. CWI Quarterly, 2:219-246, 1989.

[22] O. Müller. I/O automata and beyond: Temporal logic and abstraction in Isabelle. In TPHOLs, pages 331-348, 1998.

[23] F. Pedone. Boosting system performance with optimistic distributed protocols. Computer, 34(12):80-86, 2001.

[24] A. Singh, T. Das, P. Maniatis, P. Druschel, and T. Roscoe. BFT protocols under fire. In NSDI, 2008.

[25] M. M. V. Luchangco and N. Shavit. On the uncontended complexity of consensus. In ICDCS, pages 45-59, 2003.

[26] M. Wenzel. Isar - a generic interpretative approach to readable formal proof documents. In TPHOLs, pages 167-184, 1999. 


\section{A. Linearizability with Repeated Events}

In this section we propose a formalization of the definition of linearizability found in [12].

\section{A.1 Sequential Traces}

A sequential trace in $\operatorname{sig}_{T}$ is a trace in $\operatorname{sig}_{T}$ composed of alternating invocation and responses, starting with an invocation, and such that a response at index $i$ responds to the invocation at index $i-1$.

Definition 37. Sequential Trace. A trace $t$ in $\operatorname{sig}_{T}$ is sequential iff $t(1)=\operatorname{inv}\left({ }_{-},,_{-}\right)$and for all $i \in[1 . .|t|-1]$, if $t(i)=\operatorname{inv}\left(c,_{-}\right.$, in $)$ then $t(i+1)=\operatorname{res}\left(c,{ }_{-}\right.$, in, $\left.{ }_{-}\right)$.

Example 3. A sequential trace is a trace of the form

$\left[\operatorname{inv}\left(c_{1},{ }_{-}, \operatorname{in}_{1}\right), \operatorname{res}\left(c_{1},{ }_{-}\right.\right.$, in $_{1}$, out $\left._{1}\right), \operatorname{inv}\left(c_{2},{ }_{-}\right.$, in $\left._{2}\right), \operatorname{res}\left(c_{2},{ }_{-}\right.$, in $_{2}$, out $\left._{2}\right)$, $\ldots, \operatorname{inv}\left(c_{n},{ }_{-}\right.$, in $\left._{n}\right), \operatorname{res}\left(c_{n},{ }_{-}\right.$, in $_{n}$, out $\left.\left._{n}\right)\right]$

Definition 38. Trace Agreeing with an ADT. A sequential trace agrees with the ADT T iff

$$
\text { out }_{i}=f_{T}\left(\left[\mathrm{in}_{1}, \ldots, \mathrm{in}_{i}\right]\right)
$$

for all $i \in[1 . . n]$.

\section{A.2 Completion of a Trace}

Definition 39. Complete traces. A trace $t$ in sig S $_{T}$ is complete if and only if it is well-formed and it has no pending invocations.

In other words, $t$ is complete iff

- $t$ is well-formed

- for all index $i \in[1 . .|t|]$, if $t(i)=\operatorname{inv}\left({ }_{-},-\right.$, in $)$then there exists an index $j \in[i . .|t|]$ such that $t(j)=\operatorname{res}\left({ }_{-},,_{-}, i n v,,_{-}\right)$

Note that a complete trace has a number of elements that is even.

Definition 40. Completion of a trace. Trace $t^{\prime}$ is a completion of trace $t$ if and only if

- $t$ is a prefix of $t^{\prime}$

- $t^{\prime}$ is a complete trace.

\section{A.3 Linearizability of Complete Traces}

We consider a complete trace $t$ in $\operatorname{sig}_{T}$ and a sequential trace $t^{\prime}$ in $\operatorname{sig}_{T}$.

Definition 41. Reordering of a trace. Trace $t^{\prime}$ is a reordering of trace $t$ iff $|t|=\left|t^{\prime}\right|$ and there exists a permutation $\sigma$ of $[1 . .|t|]$ such that $t^{\prime}(\sigma(i))=t(i)$ for all $i \in[1 . .|t|]$.

Definition 42. Invocation index. We call invocation index any index $i \in[1 . . t]$ such that $t(i)=\operatorname{inv}\left({ }_{-},,_{-}\right)$.

Definition 43. Response function. We define the response function

$$
\operatorname{resp}_{t}:[1 . .|t|] \rightarrow[1 . .|t|]
$$

such that given an invocation index $i \in[1 . .|t|]$, if $t(i)=$ $\operatorname{inv}\left(c,{ }_{-}\right.$, in $)$then $\operatorname{resp}_{t}(i)$ is the smallest index $j \geq i$ such that $t(j)=\operatorname{res}\left(c,{ }_{-}\right.$, in,,$)$.

Definition 44. Preserving the order of non-overlapping operations. If $t^{\prime}$ is a reordering of $t$ according to $\sigma$, then trace $t^{\prime}$ preserves the ordering of non-overlapping operations in $t$ iff for all pairs $i, j$ of invocation indices,

$$
\begin{aligned}
& \text { if } \operatorname{resp}_{t}(i)<j \text { then } \sigma(i)<\sigma(j) \\
& \sigma\left(\operatorname{resp}_{t}(i)\right)=\sigma(i)+1
\end{aligned}
$$

Definition 45. Linearizable ${ }^{*}$ complete trace. A complete trace $t$ is linearizable ${ }^{*}$ iff there exists a sequential trace $t^{\prime}$ in $\operatorname{sig}_{T}$ such that:

- $t^{\prime}$ agrees with the ADT T.

- $t^{\prime}$ is a reordering of $t$.

- $t^{\prime}$ preserves the order of non-overlapping operations in $t$.
In this case we say that $t^{\prime}$ is a linearization of $t$.

\section{A.4 Linearizability*}

Definition 46. Linearizability $^{*}$ A trace $t$ in $\operatorname{sig}_{T}$ is linearizable* iff:

- Trace $t$ is well-formed

- There exists a completion of that is linearizable*.

\section{B. Equivalence Proof}

In this section we informally prove that the two definitions of linearizability given above are equivalent. If a trace $t$ is linearizable then we can use any linearization of $t$ to obtain a linearization function for $t$. Conversely, if we know a linearization function for $t$, we can obtain a linearization $t_{s e q}$ of $t$.

Consider a trace $t$ in $s i g_{T}$ that is well-formed.

Lemma 1. If a completion of trace $t$ is linearizable, then $t$ is linearizable.

Proof. Let $t_{\text {comp }}$ be a linearizable completion of trace $t$. Let $g_{\text {comp }}$ be a linearization function for $t_{c o m p}$. The restriction of $g_{c o m p}$ to the domain of $t$ is a linearization function for $t$.

Lemma 2. If $t$ is linearizable* then $t$ is linearizable.

Proof. Suppose that there exists a completion $t_{\text {comp }}$ of $t$ that is linearizable* . By definition of linearizability* we know that there exists a sequential trace $t_{s e q}$ such that:

- $t_{\text {seq }}$ agrees with the semantics of $T$.

- $t_{s e q}$ is a reordering of $t_{c o m p}$.

- $t_{\text {seq }}$ preserves the order of non-overlapping operations in $t_{c o m p}$.

The linearization function $g$. Since $t_{\text {seq }}$ agrees with the semantics of $T$ we get

$$
\begin{aligned}
& t_{\text {seq }}=\left[\operatorname{inv}\left(c_{1},{ }_{-}, \text {in }_{1}\right), \operatorname{res}\left(c_{1},{ }_{-}, \text {in }_{1}, \text { out }_{1}\right), \operatorname{inv}\left(c_{2},{ }_{-}, \text {in }_{2}\right), \operatorname{res}\left(c_{2},{ }_{-}, \text {in }_{2}, \text { out }_{2}\right),\right. \\
& \left.\ldots, \operatorname{inv}\left(c_{n},-, \operatorname{in}_{n}\right), \operatorname{res}\left(c_{n},-, \text { in }_{n}, \text { out }_{n}\right)\right] \\
& \text { with out }{ }_{i}=f_{T}\left(\left[\operatorname{in}_{1}, \mathrm{in}_{2}, \ldots, \mathrm{in}_{i}\right]\right)=f_{T}\left(\text { inputs }\left(t_{\text {seq }}, i\right)\right) \text {. }
\end{aligned}
$$

Moreover, $t_{\text {seq }}$ being a reordering of $t_{\text {comp }}$, we get that there exists a permutation $\sigma$ of $[1 . .|t|]$ such that

$$
t_{\text {seq }}(\sigma(i))=t_{\text {comp }}(i) \text { for all } i \in[1 . .|t|] .
$$

Let function $g$ be such that

$$
\text { for all commit index } i \text { of } t_{\text {comp }}, g(i)=\operatorname{inputs}\left(t_{\text {seq }}, \sigma(i)\right)
$$

In other words, $g(i)$ is the current input history at index $\sigma(i)$ in $t_{\text {seq. }}$.

We now show that $g$ is a linearization function for $t_{c o m p}$.

Function $g$ explains trance $t_{c o m p}$. If $i$ is a commit index in $t_{\text {comp }}$, then

$$
\begin{aligned}
t_{\text {comp }}(i) & =t_{\text {seq }}(\sigma(i)) & & \text { using } 5 \\
& =\operatorname{res}\left({ }_{-},{ }_{-}, f_{T}\left(\text { inputs }\left(t_{\text {seq }}, \sigma(i)\right)\right)\right. & & \text { using } 4 \\
& =\operatorname{res}\left({ }_{-},,_{-}, f_{T}(g(i))\right) & & \text { using } 6 .
\end{aligned}
$$

Hence

$$
g \text { explains trace } t_{\text {comp }} \text {. }
$$

Commit Order. For all distinct pairs $i, j$ of commit indices of $t_{c o m p}$, we get that

- $g(i)=\operatorname{inputs}\left(t_{\text {seq }}, \sigma(i)\right)$ and $g(j)=\operatorname{inputs}\left(t_{\text {seq }}, \sigma(j)\right)$, by 6 .

- $\sigma(i) \neq \sigma(j)$ and both are commit indices of $t_{\text {seq }}$, by 5 .

- inputs $\left(t_{s e q}, i\right)$ is a strict prefix of inputs $\left(t_{s e q}, j\right)$ or vice-versa, by 4 . 
Hence commit-order $\left(t_{\text {comp }}, g\right)$ holds.

It remains to show that validity $\left(t_{c o m p}, g\right)$ holds.

Validity. Consider an index $i \in\left[1 . . \mid t_{\text {comp }}\right]$ such that $t_{\text {comp }}(i)=$ $\operatorname{res}\left({ }_{-}\right.$, ,, in, _. $)$. We have that

- $t_{\text {seq }}(\sigma(i))=t_{\text {comp }}(i)$, by 5 .

- $t_{\text {seq }}(\sigma(i))=\operatorname{res}\left({ }_{-},{ }_{-}, i n^{\prime},\left[\ldots, i^{\prime}\right]\right)$, by 4 .

Hence

$$
t_{\text {comp }}(i) \text { ends with in. }
$$

It remains to show that for all commit indices $i$,

$$
\operatorname{elems}(g(i)) \subseteq \operatorname{elems}\left(\operatorname{inputs}\left(t_{c o m p}, i\right)\right) \text {. }
$$

For this, suppose the there exists a commit index $i$ such that

$$
\text { elems }(g(i)) \nsubseteq \text { elems }\left(\operatorname{inputs}\left(t_{\text {comp }}, i\right)\right) \text {. }
$$

Then we know that there exits an invocation index $j$ such that

$$
\begin{aligned}
& j>i \\
& \sigma(j)<\sigma(i)-1
\end{aligned}
$$

Let the invocation index $k$ be such that

$$
\operatorname{resp}_{t_{\text {comp }}}(k)=i \text {. }
$$

From 10 and 8 we have that $\operatorname{resp}_{t_{\text {comp }}}(k)<j$, hence since $t_{\text {seq }}$ preserves the order of non-overlapping operations in $t_{\text {comp }}$ we have that

$$
\begin{aligned}
& \sigma(k)=\sigma(i)-1 \\
& \sigma(k)<\sigma(j)
\end{aligned}
$$

However from 11 and 9 we have that

$$
\sigma(j)<\sigma(k) .
$$

From $\sigma(i)<\sigma(j)$ and $\sigma(j)<\sigma(i)$ we get a contradiction. Hence for all commit index $i$,

$$
\operatorname{elems}(g(i)) \subseteq \operatorname{elems}\left(\operatorname{inputs}\left(t_{\text {comp }}, i\right)\right) .
$$

From 7 and 12 we get that

$$
\text { Validity }\left(t_{\text {comp }}, g\right) \text { holds. }
$$

Conclusion. From $g$ explains trace $t_{\text {comp }}$, commit$\operatorname{order}\left(t_{c o m p}, g\right)$, and Validity $\left(t_{c o m p}, g\right)$ we get that $g$ is a linearization function for $t_{c o m p}$. Since $t_{c o m p}$ is a completion of $t$ we get by lemma 1 that $t$ is linearizable.

Lemma 3. Suppose that $t$ is linearizable. Then there exists a completion of that is linearizable.

Proof. Suppose that $t$ admits a linearization function $g$.

Consider the last commit index $i_{\text {last }}$ of $t$. Let the history $h_{\text {last }}$ be such that $h_{\text {last }}=g\left(i_{\text {last }}\right)$. Now consider the set of all invocation indices of $t$ whose invocation is pending. Arbitrarily order this set to form the sequence is $=\left[i_{1}, i_{2}, \ldots, i_{n}\right]$. Let $c_{1} \ldots c_{n}$ and $i n_{1} \ldots i n_{n}$ be such that for all $j \in[1 . . n], t\left(i_{j}\right)=\operatorname{inv}\left(c_{j},{ }_{-}, i n_{j}\right)$.

Let $h s=\left[h_{1}, h_{2}, \ldots, h_{n}\right]$ where

$$
h_{j}=h_{j-1}:: \mathrm{in}_{j} \text { for all } j \in[2 . . n]
$$

and

$$
h_{1}=h_{\max }:: \mathrm{in}_{1}
$$

Let

$$
\begin{aligned}
t^{\prime}= & {\left[\operatorname{res}\left(c_{1},{ }_{-}, \operatorname{in}_{1}, f_{T}\left(h_{1}\right)\right), \operatorname{res}\left(c_{1},{ }_{-}, \operatorname{in}_{2}, f_{T}\left(h_{2}\right)\right),\right.} \\
& \left.\ldots, \operatorname{res}\left(c_{n},{ }_{-}, \operatorname{in}_{n}, f_{T}\left(h_{n}\right)\right)\right]
\end{aligned}
$$

and

$$
t_{c o m p}=t:: t^{\prime}
$$

Observe that $t_{c o m p}$ is a complete trace. Moreover let

$$
g_{\text {comp }}(i)=\text { if } i \leq|t| \text { then } g(i) \text { else } h_{i-|t|}
$$

for all $i \in\left[1 . .|t|+\left|t^{\prime}\right|\right]$. Observe that $g_{\text {comp }}$ is a linearization function for $t_{c o m p}$.

We conclude that $t_{\text {comp }}$ is a linearizable complete trace.

Lemma 4. Suppose that $t$ is a linearizable complete trace. Then $t$ is linearizable*.

Proof. Suppose that $t$ is a linearizable complete trace. Then $t$ is well-formed and admits a linearization function $g$. Function $g$ is such that

- Function $g$ explains $t$.

- The validity $(t, g)$ predicate holds.

- The commit-order $(t, g)$ predicate holds

Consider a permutation $\sigma$ of $[1 . .|t|]$ such that:

- For all distinct commit index $i$ and $j$, if $g(i)$ is a strict prefix of $g(j)$ then $\sigma(i)<\sigma(j)$.

- For all invocation index $i$ of $t, \sigma(i)=\sigma\left(\operatorname{resp}_{t}(i)\right)-1$.

Let the trace $t_{\text {seq }}$ be such that $\left|t_{\text {seq }}\right|=|t|$ and for all $i \in[1 . .|t|]$, $t(i)=t_{s} e q \sigma(i)$. Observe that $t_{s e q}$ is a sequential trace. Hence to prove that $t$ is linearizable* it remains to show that $t_{s e q}$ agrees with the ADT $T$. Because $g$ is a linearization function for $t$ we know that if $t(i)=\operatorname{res}\left({ }_{-},,_{-}\right.$, out $)$then out $=f_{T}(g(i))$. Hence if $t_{\text {seq }}(i)=\operatorname{res}\left({ }_{-},{ }_{-}\right.$, , out $)$then out $=f_{T}\left(g\left(\sigma^{-1}(i)\right)\right)$. Moreover, from validity $(t, g)$ we get that $g(i)=\operatorname{inputs}\left(t_{\text {seq }}, \sigma(i)\right)$. Therefore $t_{\text {seq }}$ agrees with the ADT $T$.

Lemma 5. Suppose that $t$ is linearizable. Then there exists a completion of that is linearizable*.

Proof. By lemma 3 and 4.

Theorem 4. A trace t in sig s $_{T}$ inearizable iff it is linearizable*.

Proof. By lemma 2 and 5

\section{Composition Theorem}

The composition theorem states that the composition of two speculation phases is a speculation phase.

Theorem 5. Suppose that

$$
S_{1}=\operatorname{SLin}_{T}(m, n)
$$

and

Then

$$
S_{2} \models \operatorname{Sin}_{T}(n, o)
$$

$$
\operatorname{proj}\left(S_{1} \| S_{2}, \operatorname{sig}_{T}(m, o, \text { Init })\right) \models \operatorname{SLin}_{T}(m, o) .
$$

In the rest of this section we prove theorem 5 .

We consider an ADT $T$ and two speculation phases $\left(m, n\right.$, Init, $\left.r_{\text {init }}\right)$ and $(n, o)$ and such that $m<n<o$, Init $\neq \emptyset$, and $r_{\text {init }} \neq \emptyset$.

Consider a trace

$$
t \in \operatorname{acts}\left(\operatorname{sig}_{T}(m, o, \text { Init })\right)^{*}
$$

and let

and

$$
t_{m n}=\operatorname{proj}\left(t, \operatorname{acts}\left(\operatorname{sig}_{T}(m, n, \text { Init })\right)\right)
$$

$$
t_{n o}=\operatorname{proj}\left(t, \operatorname{acts}\left(\operatorname{sig}_{T}(n, o, \text { Init })\right)\right) .
$$

Assume that $t_{m n}$ is $(m, n)$-abortable linearizable and that $t_{n o}$ is $(n, o)$-abortable linearizable.

We would like to show that 
- The trace $t$ is $(m, o)$-well formed.

- For all interpretation $f_{\text {init }}$ of the init actions of $t$, there exists two functions $g:[1 . .|t|] \rightarrow I_{T}^{*}$ and $f_{\text {abort }}:[1 . .|t|] \rightarrow I_{T}^{*}$ such that:

- $f_{\text {abort }}$ is an interpretation of the abort actions of $t$.

- $g$ is an $\left(f_{\text {init }}, f_{\text {abort }}, m, n\right)$-abortable linearization function for $t$.

First observe that

$\operatorname{acts}\left(\operatorname{sig}_{T}(m, n\right.$, Init $\left.)\right) \cup \operatorname{acts}\left(\operatorname{sig}_{T}(n, o\right.$, Init $\left.)\right)=\operatorname{acts}\left(\operatorname{sig}_{T}(m, o\right.$, Init $\left.)\right)$.

Hence all actions of $t$ appear at least in one of $t_{m n}$ or $t_{n o}$.

We define two injective functions $\operatorname{pos}_{m n}^{\prime}:\left[1 . .\left|t_{m n}\right|\right] \rightarrow[1 . .|t|]$ and $\operatorname{pos}_{n o}^{\prime}:\left[1 . .\left|t_{n o}\right|\right] \rightarrow[1 . .|t|]$ that create a correspondence between the indices of $t$ and its two sub-traces $t_{m n}$ and $t_{n o}$.

The function $\operatorname{pos}_{m n}^{\prime}$ is such that for all $i \in\left[1 . .\left|t_{m n}\right|\right]$ :

- $t_{m n}(i)=t\left(\operatorname{pos}_{m n}^{\prime}(i)\right)$

- $\operatorname{pos}_{m n}^{\prime}$ is strictly monotonically increasing.

Similariy the function $\operatorname{pos}_{n o}^{\prime}$ is such that for all $i \in\left[1 . .\left|t_{n o}\right|\right]$ :

- $t_{n o}(i)=t\left(\operatorname{pos}_{n o}^{\prime}(i)\right)$.

- $\operatorname{pos}_{n o}^{\prime}$ is strictly monotonically increasing.

We now define the two functions $\operatorname{pos}_{m n}=\left(\operatorname{pos}_{m n}^{\prime}\right)^{-1}$ and $\operatorname{pos}_{n o}=\left(\operatorname{pos}_{n o}^{\prime}\right)^{-1}$. Note that $\operatorname{pos}_{m n}$ and $\operatorname{pos}_{n o}$ are injective partial functions. Moreover, for any $i \in[1 . . \mid t], i$ is in the domain of at least one of $\operatorname{pos}_{m n}$ or $\operatorname{pos}_{n o}$.

We will be able to relate properties of $t_{m n}$ and $t_{n o}$ thanks to lemma 6. Informally, lemma 6 says that the abort histories of speculation phase $(m, n)$ are the init histories of speculation phase $(n, o)$.

Lemma 6. For all index $i \in[1 . .|t|]$, the index $\operatorname{pos}_{m n}(i)$ is a $\left(t_{m n}, m, n\right)$-abort index if and only if the index $\operatorname{pos}_{n o}(i)$ is a $\left(t_{n o}, n, o\right)$-init index.

Proof. By the definition of $\left(t_{m n}, m, n\right)$-abort index and $\left(t_{n o}, n, o\right)$-init index.

\section{C.1 Trace $t$ is Well-Formed.}

We now prove that if $t_{m n}$ and $t_{n o}$ are both well-formed then $t$ is well-formed.

Lemma 7. For all client $c$, if $\operatorname{sub}\left(t_{m n}, c\right.$, Init $)$ is $(m, n)$ well-formed and $s u b\left(t_{n o}, c\right.$, Init) is $(n, o)$-well-formed then sub(t, $c$, Init) is $(m, o)$-well-formed.

Proof. Consider a client $c$. Let $t_{m n}^{c}=s u b\left(t_{m n}, c\right.$, Init $), t_{n o}^{c}=$ $\operatorname{sub}\left(t_{n o}, c\right.$, Init $)$, and $t^{c}=\operatorname{sub}(t, c$, Init) be the client projections of $t_{m n}, t_{n o}$, and $t$. Let us consider the two following cases.

Suppose that there exists no $\left(t_{m n}^{c}, m, n\right)$-abort index. Then by lemma 6 there exists no $\left(t_{n o}^{c}, n, o\right)$-init index. Then because $t_{n o}^{c}$ is $(n, o)$-well-formed we have that $t_{n o}^{c}=[]$. Thus $t^{c}=t_{m n}^{c}$. Because $t_{m n}^{c}$ is $(m, n)$-well-formed and $n>1$ we get that $t^{c}$ is $(m, o)$-wellformed.

Now we assume that there exists a $\left(t_{m n}^{c}, m, n\right)$-abort index $i_{\text {abort }}$. Let us define the strictly monotically increasing functions $\operatorname{pos}_{m n}^{c}$ and $\operatorname{pos}_{n o}^{c}$ such that, for all $i \in\left[1 . .\left|t^{c}\right|\right]$,

- If $t^{c}(i) \in \operatorname{sig}_{T}\left(c, m, n\right.$, Init) then $t^{c}(i)=t_{m n}^{c}\left(\operatorname{pos}_{m n}^{c}(i)\right)$.

- If $t^{c}(i) \in \operatorname{sig}_{T}\left(c, n, o\right.$, Init) then $t^{c}(i)=t_{n o}^{c}\left(\operatorname{pos}_{n o}^{c}(i)\right)$.

By analogy with lemma 6 we get that for all index $i \in\left[1 . .\left|t^{c}\right|\right]$, the index $\operatorname{pos}_{m n}^{c}(i)$ is a $\left(t_{m n}^{c}, m, n\right)$-abort index if and only if the index $\operatorname{pos}_{n o}^{c}(i)$ is a $\left(t_{n o}^{c}, n, o\right)$-init index.

Let $j=\left(\operatorname{pos}_{m n}^{c}\right)^{-1}\left(i_{\text {abort }}\right)$. Because $t_{m n}$ is $(m, n)$-valid with have that $i_{\text {abort }}=\left|t_{m n}^{c}\right|$. Let $k=\operatorname{pos}_{n o}^{c}(j)$. By the analogous to lemma 6 we know that $k$ is a $\left(t_{n o}^{c}, n, o\right)$-init index. Because $t_{n o}^{c}$ is $(n, o)$-well-formed we know that $j=1$.

To sum up we have obtained an index $j \in\left[1 . .\left|t^{c}\right|\right]$ such that $\operatorname{pos}_{m n}^{c}(j)$ is the last index of $t_{m n}^{c}$ and $\operatorname{pos}_{n o}^{c}(j)$ is the first index of $t_{n o}^{c}$. Thus $t^{c}$ is the concatenation of $t_{m n}^{c}$ and $t_{n o}^{c}$. It is then easy to see that $t^{c}$ is $(m, o)$-well formed because $t_{m n}^{c}$ is $(m, n)$-well formed and $t_{n o}^{c}$ is $(n, o)$-well formed.

Now consider a function $f_{\text {init }}$ that is an interpretation of the init actions in $t$.

C.2 Existence of $f_{\text {abort }}$ and $g$ such that $g$ is an $\left(f_{\text {init }}, f_{\text {abort }}, m, o\right.$ )-abortable linearization function.

Because $t_{m n}$ is $(m, n)$-abortable linearizable we know that there exists $g_{m n}$ and $f_{\text {abort }}^{m n}$ such that

- $f_{\text {abort }}^{m n}$ is an interpretation of the abort actions of $t_{m n}$.

- $g_{m n}$ is an $\left(f_{\text {init }}, f_{\text {abort }}^{m n}, m, n\right)$-abortable linearization function for $t_{m n}$.

Let $f_{\text {init }}^{n o}=f_{\text {abort }}^{m n} \circ \operatorname{pos}_{m n} \circ\left(\text { pos }_{n o}\right)^{-1}$. By lemma 6 we have that $f_{\text {init }}^{n o}$ is an interpretation of the init actions of $t_{n o}$.

Hence, because $t_{n o}$ is $(n, o)$-abortable linearizable we know that there exists $g_{n o}$ and $f_{\text {abort }}^{n o}$ such that

- $f_{\text {abort }}^{n o}$ is an interpretation of the abort actions of $t_{n o}$.

- $g_{n o}$ is an $\left(f_{\text {abort }}^{m n}, f_{\text {abort }}^{n o}, n, o\right)$-abortable linearization function for $t_{n o}$.

Let the function $g$ be such that, for all commit index $i$ of $t$,

- if $i$ is a $(t, m, n)$-commit index then $g(i)=g_{m n}\left(\operatorname{pos}_{m n}(i)\right)$.

- if $i$ is a $(t, n, o)$-commit index then $g(i)=g_{n o}\left(\operatorname{pos}_{n o}(i)\right)$.

Let $f_{\text {abort }}=f_{\text {abort }}^{\text {no }}$.

We will show that:

- $g$ explains trace $t$.

- The following predicates are satisfied:

- Validity $\left(t, g, f_{\text {init }}, f_{\text {abort }}\right)$.

- Commit-Order $(t, g)$.

- Init-Order $\left(t, g, f_{\text {init }}, f_{\text {abort }}\right)$.

- Abort-Order $\left(t, g, f_{\text {abort }}\right)$.

Lemma 8. The function g explains trace $t$.

Proof. By the definition of $g$ and the fact that $g_{m n}$ explains trace $t_{m n}$ and $g_{n o}$ explains trace $t_{n o}$.

Since $g_{m n}$ is an an $\left(f_{\text {init }}, f_{\text {abort }}^{m n}, m, n\right)$-abortable linearization function for $t_{m n}$ and $g_{n o}$ is an $\left(f_{\text {init }}^{n o}, f_{\text {abort }}^{n o}, n, o\right)$-abortable linearization function for $t_{n o}$ we know that the following predicates are satisfied:

- Validity $\left(t_{m n}, g_{m n}, f_{\text {init }}, f_{\text {abort }}^{m n}\right)$

- Commit-Order $\left(t_{m n}, g_{m n}\right)$

- Init-Order $\left(t_{m n}, g_{m n}, f_{\text {init }}, f_{\text {abort }}^{m n}\right)$

- Abort-Order $\left(t_{m n}, g_{m n}, f_{\text {abort }}^{m n}\right)$

- Validity $\left(t_{n o}, g_{n o}, f_{\text {init }}^{n o}, f_{\text {abort }}^{n o}\right)$

- Commit-Order $\left(t_{n o}, g_{n o}\right)$

- Init-Order $\left(t_{n o}, g_{n o}, f_{\text {init }}^{n o}, f_{\text {abort }}^{n o}\right)$

- Abort-Order $\left(t_{n o}, g_{n o}, f_{\text {abort }}^{n o}\right)$

Lemma 9. Validity $\left(t, g, f_{\text {init }}, f_{\text {abort }}\right)$ holds. 
Proof. We first show that any commit index of $t$ is $\left(t, g, f_{\text {init }}\right)$-valid. Consider an index $i \in[1 . .|t|]$ such that $t(i)=\operatorname{res}\left({ }_{-},,_{-}\right.$, in,,,$\left._{-}\right)$. We would like to show that

- $\operatorname{elems}(g(i)) \subseteq v i\left(m, t, f_{\text {init }}, i\right)$

- The input in is the last element of $g(i)$.

Observe that either $i$ is a $(t, m, n)$-commit index or $i$ is a $(t, n, o)$-commit index.

Suppose that $i$ is a $(t, m, n)$-commit index. It follows that:

1. $t(i)=t_{m n}\left(\operatorname{pos}_{m n}(i)\right)$, by definition of $\operatorname{pos}_{m n}$

2. $g(i)=g_{m n}\left(\operatorname{pos}_{m n}(i)\right)$, by definition of $g$

3. The index $\operatorname{pos}_{m n}(i)$ is $\left(t_{m n}, g_{m n}, f_{\text {init }}\right)$-valid, by Validity $\left(t_{m n}, g_{m n}, f_{\text {init }}, f_{\text {abort }}^{m n}\right)$

From 1 and 3 we get that the input in is the last element of $g_{m n}(i)$. With 2 we conclude that the input in is the last element of $g(i)$. It remains to show that elems $(g(i)) \subseteq v i\left(m, t, f_{\text {init }}, i\right)$

From 3 we have that elems $\left(g_{m n}(i)\right)$ $v i\left(m, t_{m n}, f_{\text {init }}, \operatorname{pos}_{m n}(i)\right)$. With 2 we get that elems $(g(i)) \subseteq$ $v i\left(m, t_{m n}, f_{\text {init }}, \operatorname{pos}_{m n}(i)\right)$. From the fact that $t_{m n}$ is a subtrace of $t$ we get that

- elems(inputs $\left.\left(t_{m n}, \operatorname{pos}_{m n}(i)\right)\right) \subseteq \operatorname{elems}($ inputs $(t, i))$.

- $i v i\left(m, t_{m n}, f_{\text {init }}, \operatorname{pos}_{m n}(i)\right) \subseteq i v i\left(m, t, f_{\text {init }}, i\right)$.

Hence $v i\left(m, t_{m n}, f_{\text {init }}, \operatorname{pos}_{m n}(i)\right) \subseteq v i\left(m, t, f_{\text {init }}, i\right)$. Finally we get that elems $(g(i)) \subseteq v i\left(m, t, f_{\text {init }}, i\right)$.

Now suppose that $i$ is a $(t, n, o)$-commit index. It follows that:

1. $t(i)=t_{n o}\left(\operatorname{pos}_{n o}(i)\right)$, by definition of $\operatorname{pos}_{n o}$

2. $g(i)=g_{\text {no }}\left(\operatorname{pos}_{n o}(i)\right)$, by definition of $g$

3. The index $\operatorname{pos}_{n o}(i)$ is $\left(t_{n o}, g_{n o}, f_{\text {abort }}^{m n}\right)$-valid, by Validity $\left(t_{n o}, g_{n o}, f_{\text {init }}^{n o}, f_{\text {abort }}^{\text {no }}\right)$

As before we get that input in is the last element of $g(i)$. It remains to show that elems $(g(i)) \subseteq v i\left(m, t, f_{\text {init }}, i\right)$.

From 3 we have that elems $\left(g_{\text {no }}(i)\right)$ $v i\left(n, t_{n o}, f_{\text {init }}^{n o}, \operatorname{pos}_{n o}(i)\right)$. With 2 we get that elems $(g(i)) \subseteq$ $v i\left(n, t_{n o}, f_{\text {abort }}^{m n}, \operatorname{pos}_{n o}(i)\right)$.

By definition we have that

$$
\begin{aligned}
& v i\left(n, t_{n o}, f_{\text {abort }}^{m n}, \operatorname{pos}_{n o}(i)\right)= \\
& \quad i v i\left(n, t_{n o}, f_{\text {abort }}^{m n}, \operatorname{pos}_{n o}(i)\right) \cup \operatorname{elems}\left(\operatorname{inputs}\left(t_{n o}, \operatorname{pos}_{n o}(i)\right)\right) .
\end{aligned}
$$

By lemma 6 we have that

$$
\begin{aligned}
& i v i\left(n, t_{n o}, f_{\text {abort }}^{m n}, \operatorname{pos}_{n o}(i)\right)= \\
& \bigcup\left\{\text { elems }\left(f_{\text {abort }}^{m n}(v)\right) \cup\{\text { in }\} \mid\right. \\
& \left.\quad \exists j \in\left[1 . .\left|t_{m n}\right|\right] . t_{m n}(j)=\operatorname{swi}(-, n, \text { in }, v)\right\} .
\end{aligned}
$$

Moreover from Validity $\left(t_{m n}, g_{m n}, f_{\text {init }}, f_{\text {abort }}^{m n}\right)$ we have that, for all indices $\left.j \in\left[1 . .\left|t_{m n}\right|\right]\right]$ such that there exists in and $v$ where $t_{m n}(j)=\operatorname{swi}\left({ }_{-}, n\right.$, in, $\left.v\right)$,

$$
\text { elems }\left(f_{\text {abort }}^{m n}(v)\right) \cup\{\text { in }\} \subseteq v i\left(m, t_{m n}, f_{\text {init }}, j\right) .
$$

Hence, by properties of the multiset union, we have that

$$
i v i\left(n, t_{n o}, f_{\text {abort }}^{m n}, \operatorname{pos}_{n o}(i)\right) \subseteq v i\left(m, t_{m n}, f_{\text {init }}, \operatorname{pos}_{m n}(i)\right) .
$$

Hence we have that

$$
\begin{aligned}
& v i\left(n, t_{n o}, f_{\text {abort }}^{m n}, \operatorname{pos}_{n o}(i)\right) \subseteq \\
& \quad v i\left(m, t_{m n}, f_{\text {init }}, \operatorname{pos}_{m n}(i)\right) \cup \operatorname{elems}\left(\text { inputs }\left(t_{n o}, \operatorname{pos}_{n o}(i)\right)\right) .
\end{aligned}
$$

Moreover,

$$
\begin{aligned}
& v i\left(m, t_{m n}, f_{\text {init }}, \operatorname{pos}_{m n}(i)\right) \cup \text { elems }\left(\text { inputs }\left(t_{n o}, \operatorname{pos}_{n o}(i)\right)\right)= \\
& \quad v i\left(m, t, f_{\text {init }}, i\right) \cup \text { elems }(\text { inputs }(t, i))
\end{aligned}
$$

Thus $v i\left(n, t_{n o}, f_{\text {abort }}^{m n}, p_{\text {nos }}(i)\right) \subseteq \quad v i\left(m, t, f_{\text {init }}, i\right)$. With elems $(g(i)) \subseteq v i\left(n, t_{n o}, f_{a b o r t}^{m n}, \operatorname{pos}_{n o}(i)\right)$ we conclude that elems $(g(i)) \subseteq v i\left(n, t, f_{\text {init }}, i\right)$.

By a similar reasoning we can show that any abort index of $t$ is $\left.\left(t, f_{\text {init }}, f_{\text {abort }}\right)\right)$-valid.

Lemma 10. Commit-Order $(t, g)$ holds.

Proof. Consider any two $(t, g)$-commit histories $h_{1}$ and $h_{2}$. Suppose that both $h_{1}$ and $h_{2}$ appear in $t_{m n}$. Then by Commit$\operatorname{Order}\left(t_{m n}, g_{m n}\right)$ we have that one is the prefix of the other. Suppose that both $h_{1}$ and $h_{2}$ appear in $t_{n o}$. Then by Commit$\operatorname{Order}\left(t_{n o}, g_{n o}\right)$ we have that one is the prefix of the other.

Suppose that $h_{1}$ appears in $t_{m n}$ and that $h_{2}$ appears in $t_{n o}$. By Init-Order $\left(t_{n o}, g_{n o}, f_{\text {init }}^{n o}, f_{\text {abort }}^{\text {no }}\right)$ and we know that there exists an index $j$ such that $\operatorname{pos}_{n o}(j)$ is a $\left(t_{n o}, n, o\right)$-init index and $h^{\prime}=f_{\text {init }}^{\text {no }}\left(\operatorname{pos}_{n o}(j)\right)$ is a prefix of $h_{2}$. By lemma 6 we have that $\operatorname{pos}_{m n}(j)$ is a $\left(t_{m n}, m, n\right)$-abort index and $h^{\prime}=f_{\text {abort }}^{m n}\left(\operatorname{pos}_{m n}(j)\right)$. Hence by Abort-Order $\left(t_{m n}, g_{m n}, f_{\text {init }}\right)$ we get that $h_{1}$ is a prefix of $h^{\prime}$. By transitivity we get that $h_{1}$ is a prefix of $h_{2}$.

The case where $h_{2}$ appears in $t_{m n}$ and that $h_{1}$ appears in $t_{n o}$ is very similar.

Lemma 11. Init-Order $\left(t, g, f_{\text {init }}, f_{\text {abort }}^{n o}\right)$ holds.

Proof. Let $h_{\text {init }}^{\text {mo }}$ be the longest common prefix of all $\left(t, m, o, f_{\text {init }}\right)$ init histories. Observe that $h_{\text {init }}$ is also the longest common prefix of all $\left(t_{m n}, m, n, f_{\text {init }}\right)$-init histories. We first show that $h_{i n i t}^{\text {mo }}$ is a prefix of any $(t, g)$-commit history. Consider a $(t, g)$-commit history $h$. Observe that $h$ is either a $\left(t_{m n}, g_{m n}\right)$-commit history or a $\left(t_{n o}, g_{n o}\right)$-commit history.

Suppose that $h$ is a $\left(t_{m n}, g_{m n}\right)$-commit history. Since $h_{i n i t}^{m o}$ is the longest common prefix of all $\left(t_{m n}, m, n\right)$-init histories we have that $h_{\text {init }}^{m o}$ is a prefix of $h$ by Init-Order $\left(t_{m n}, g_{m n}, f_{\text {init }}, f_{\text {abort }}^{m n}\right)$.

Suppose that $h$ is a $\left(t_{n o}, g_{n o}\right)$-commit history. Let $h_{\text {init }}^{n o}$ be the longest common prefix of all $\left(t_{n o}, n, o\right)$-init histories. By Init$\operatorname{Order}\left(t_{n o}, g_{n o}, f_{\text {init }}^{n o}, f_{\text {abort }}^{n o}\right)$ we have that $h_{\text {init }}^{\text {no }}$ is a prefix of $h$.

Moreover by lemma 6 we have that $h_{\text {init }}^{\text {no }}$ is the longest common prefix of all $\left(t_{m n}, m, n\right)$-abort histories. But by

Init-Order $\left(t_{m n}, g_{m n}, f_{\text {init }}, f_{\text {abort }}^{m n}\right)$ we have that $h_{i n i t}^{m o}$, which is equal to $h_{i n i t}^{m n}$, is a prefix of any $\left(t_{m n}, m, n\right)$-abort history. Since $h_{i n i t}^{n o}$ is the longest common prefix of all $\left(t_{m n}, m, n\right)$-abort histories we get that $h_{\text {init }}^{\text {mo }}$ is a prefix of $h_{i n i t}^{\text {no }}$.

History $h_{\text {init }}^{\text {no }}$ being a prefix of $h$ and history $h_{\text {init }}^{\text {mo }}$ being a prefix of $h_{i n i t}^{\text {no }}$, we get that $h_{\text {init }}^{\text {mo }}$ is a prefix of $h$.

We can prove by a similar reasoning that $h_{\text {init }}^{\text {mo }}$ is a prefix of any $\left(t, m, o, f_{\text {abort }}^{n o}\right)$-abort history.

Lemma 12. Abort-Order $\left(t, g, f_{\text {abort }}^{\text {no }}\right)$ holds.

Proof. Consider a $(t, g)$-commit history $h$ and a $\left(t, m, o, f_{\text {abort }}^{n o}\right)$ abort history $h^{\prime}$. Observe that $h^{\prime}$ is also a $\left(t_{n o}, n, o, f_{\text {abort }}^{n o}\right)$-abort history. Observe that $h$ is either a $\left(t_{m n}, g_{m n}\right)$-commit history or a $\left(t_{n o}, g_{n o}\right)$-commit history.

Suppose that $h$ is a $\left(t_{n o}, g_{n o}\right)$-commit history. Since $h^{\prime}$ is a $\left(t_{n o}, n, o, f_{\text {abort }}^{n o}\right)$-abort history we get by Abort$\operatorname{Order}\left(t_{n o}, g_{n o}, f_{\text {abort }}^{n o}\right)$ that $h$ is a prefix of $h^{\prime}$.

Suppose that $h$ is a $\left(t_{m n}, g_{m n}\right)$-commit history. By Abort$\operatorname{Order}\left(t_{m n}, g_{m n}, f_{\text {abort }}^{m n}\right)$ we have that $h$ is a prefix of any $\left(t_{m n}, m, n, f_{\text {abort }}^{m n}\right)$-abort history. Hence $h$ is a prefix of the longest common prefix of all $\left(t_{m n}, m, n, f_{\text {abort }} m n\right)$-abort histories. Hence by lemma 6 we have that $h$ is a prefix of the longest common prefix of all $\left(t_{n o}, n, o, f_{\text {abort }} m n\right)$-init histories, noted $h_{i n i t}^{n o}$. Moreover by Init-Order $\left(t_{n o}, g_{n o}, f_{\text {init }}^{n o}, f_{\text {abort }}^{n o}\right)$ we have that $h_{\text {init }}^{n o}$ is a prefix of any $\left(t, m, o, f_{\text {abort }}^{n o}\right)$-abort history. Hence $h_{\text {init }}^{n o}$ is a prefix of $h^{\prime}$.

In conclusion, $h$ being a prefix of $h_{i n i t}^{n o}$ and $h_{i n i t}^{\text {no }}$ being a prefix of $h^{\prime}$, we get that $h$ is a prefix of $h^{\prime}$. 
Lemma 13. Suppose that trace $t_{m n}$ is $(m, n)$-abortably linearizable and that trace $t_{n o}$ is $(n, o)$-abortably linearizable. Then trace $t$ is $(m, o)$-abortably linearizable.

Proof. From lemma 7 we get that $t$ is $(m, o)$-well-formed.

For all interpretation $f_{\text {init }}$ of the init actions of $t$, we get from lemmas $8,9,10$, and 12 that there exit a function $g$ and an interpretation $f_{\text {abort }}$ of the abort actions of $t$ such that $g$ explains trace $t$ and such that the following predicates are satisfied:

- $\operatorname{Validity}\left(t, g, f_{\text {init }}, f_{\text {abort }}\right)$

- Commit-Order $(t, g)$

- Init-Order $\left(t, g, f_{\text {init }}, f_{\text {abort }}\right)$

- $\operatorname{Abort-Order}\left(t, g, f_{\text {abort }}\right)$

We conclude that $t$ is $(m, o)$-abortably linearizable. 